\title{
Aberrant Fluid Shear Stress Contributes to Articular Cartilage Pathogenesis via Epigenetic Regulation of ZBTB20 by H3K4me3
}

\author{
Yu Jin* \\ Zhenxia Li* \\ Yanran Wu \\ Hairui Li \\ Zhen Liu \\ Lu Liu \\ Ningjuan Ouyang \\ Ting Zhou \\ Bing Fang \\ Lunguo Xia
}

Department of Orthodontics, Shanghai Ninth People's Hospital, Shanghai Jiao Tong University School of Medicine; College of Stomatology, Shanghai Jiao Tong University; National Center for Stomatology; National Clinical Research Center for Oral Diseases, Shanghai Key Laboratory of Stomatology, Shanghai, 2000 I I, People's Republic of China

*These authors contributed equally to this work

\begin{abstract}
Purpose: Osteoarthritis (OA) is a common disease for human beings, characterized by severe inflammation, cartilage degradation, and subchondral bone destruction. However, current therapies are limited to relieving pain or joint replacement and no effective treatment methods have been discovered to improve degenerative changes. Currently, a variety of evidences have indicated that aberrant mechanical stimuli is closely associated with articular joint pathogenesis, while the detailed underlying mechanism remains unelucidated. In the present study, we determined to investigate the impact of excessive high fluid shear stress (FSS) on primary chondrocytes and the underlying epigenetic mechanisms.
\end{abstract}

Materials and Methods: Phalloidin staining and EdU staining were used to evaluate cell morphology and viability. The mRNA level and protein level of genes were determined by qPCR, Western blot assay, and immunofluorescence staining. Mechanistic investigation was performed through RNA-sequencing and CUT\&Tag sequencing. In vivo, we adopted unilateral anterior crossbites (UAC) mice model to investigate the expression of $\mathrm{H} 3 \mathrm{~K} 4 \mathrm{me} 3$ and ZBTB20 in aberrant force-related cartilage pathogenesis.

Results: The results demonstrated that FSS greatly disrupts cell morphology and significantly decreased chondrocyte viability. Aberrant FSS induces remarkable inflammatory mediators production, leading to cartilage degeneration and degradation. In depth mechanistic study showed that FSS results in more than 10-fold upregulation of H3K4me3, and the modulatory effect of H3K4me3 on cartilage was obtained by directly targeting ZBTB20. Furthermore, Wnt signaling was strongly activated in high FSS-induced OA pathogenesis, and the negative impact of ZBTB20 on chondrocytes was also achieved through activating Wnt signaling pathway. Moreover, pharmacological inhibition of H3K4me3 activation by MM-102 or treatment with Wnt pathway inhibitor LF3 could effectively alleviate the destructive effect of FSS on chondrocytes. In vivo UAC mice model validated the dysregulation of H3K4me3 and ZBTB20 in aberrant force-induced cartilage pathogenesis.

Conclusion: Through the combination of in vitro FSS model and in vivo UAC model, KMT2B-H3K4me3-ZBTB20 axis was first identified in aberrant FSS-induced cartilage pathogenesis, which may provide evidences for epigenetic-based therapy in the future.

Keywords: osteoarthritis, fluid shear stress, $\mathrm{H} 3 \mathrm{~K} 4 \mathrm{me} 3$, epigenetic, cartilage degeneration

\section{Introduction}

Osteoarthritis (OA) is a prevalent degenerative joint disease which severely impairs the life quality of human beings. Pathologically, OA is a complex disorder which may involve cartilage degradation, synovial inflammation, subchondral bone sclerosis, infrapatellar fat pad fibrosis, and meniscal damage. ${ }^{1,2}$ However, due to the lack 
of comprehensive understanding of the underlying mechanism during OA progression, current therapies are merely limited to relieving symptoms or slowing down the disease progression and joint replacement surgery at the end stage is finally applied. ${ }^{3}$ Therefore, it is of critical significance to clarify the potential mechanism of OA pathogenesis to discover innovative therapy methods.

As a site biomechanically related to external forces, articular cartilage is frequently associated with $\mathrm{OA}$ and mechanical loading is determined as a crucial factor on the onset and progression of cartilage pathogenesis. ${ }^{4}$ Physiologically, chondrocytes in articular cartilage are subjected to a variety of stresses, strains, and pressure that come from daily activities. ${ }^{5}$ However, in some cases, abnormal mechanical overloading of cartilage could lead to chondrocyte apoptosis, extracellular matrix degeneration, resulting in OA initiation and progression. ${ }^{6-8}$ Among the different kinds of mechanical forces, fluid shear stress (FSS) serves as an essential physiological and pathological factor for articular joint. ${ }^{9,10}$ It was indicated that relative low shear stress $\left(<5 \mathrm{dyne} / \mathrm{cm}^{2}\right)$ is chondroprotective, whereas high shear stress (10-20 dyne $\left./ \mathrm{cm}^{2}\right)$ induces inflammatory responses, promotes matrix-degrading enzymes expression, consequently results in matrix degradation and OA-like pathological features. ${ }^{5,8,11}$ Due to the limited capacity of cartilage for tissue repair and regeneration, ${ }^{12}$ investigation of potential mechanisms underlying FSS-induced OA pathogenesis is greatly needed.

In recent years, more and more attention has focused on epigenetic alterations, which were demonstrated to mediate various kinds of pathological responses. Various kinds of histones modifications, including methylation, phosphorylation and acetylation, regulate key genes which participate in disease initiation and progression. ${ }^{13}$ For example, it was reported that elevated NSD3 histone methylation activity drives the malignant progression of squamous cell lung cancer and indicates the promising prospect of NSD3-dependent therapy strategy. ${ }^{14} \mathrm{OA}$ is a common disease which has gained increasingly more attention and studied by many researchers. A recent study has reported an abnormal expression of histone deacetylases (HDAC) in OA pathogenesis, implying the prospect of treating $\mathrm{OA}$ through regulating specific HDACs activity. ${ }^{15,16}$ Another study demonstrated the importance of EZH2 in OA, providing evidences for epigenetic modifications during OA development. ${ }^{17}$ Specifically, the data indicated that EZH2 may suppress
OA progression through inhibiting miR-138 expression by promoting the histone methylation of its promoter. Although several findings have suggested the potential functions of epigenetics on OA pathogenesis, ${ }^{18,19}$ the detailed regulatory mechanism and the underlying epigenetic-based therapeutic target have not been discovered until now. More importantly, the specific mechanism of epigenetic regulation involved in mechanical force-related OA pathogenesis remains elusive.

In the current study, we determined to identify the effect of aberrant FSS on primary chondrocytes and illuminate the involvement of $\mathrm{H} 3 \mathrm{~K} 4 \mathrm{me} 3$ in aberrant FSSinduced OA. Through in vitro FSS model and in vivo unilateral anterior crossbites (UAC) mice model, we confirmed the impact of aberrant FSS on osteoarthritis-like pathological changes in joint regions. Moreover, we aimed to elucidate the involvement of epigenetic regulation in mechanical-related cartilage pathogenesis and to investigate whether targeting epigenetic event may serve as promising therapeutic strategy in the future.

\section{Materials and Methods}

\section{Ethics}

This study was approved by the Ethics Committee of Shanghai Jiao Tong University. All the animal studies were approved by the Shanghai Jiao Tong University Institute Animal Care and Use Committee, and conducted according to the Guide for Care and Use of Laboratory Animals (Ministry of Science and Technology of China, 2006).

\section{Primary Chondrocytes Isolation and Culture}

Chondrocytes were obtained from the articular cartilage of 3-week-old male C57BL/6J mice. Specifically, obtained cartilage was digested in $0.25 \%$ trypsin-EDTA in $37^{\circ} \mathrm{C}$ for $0.5 \mathrm{~h}$. After being cut into approximately $1 \mathrm{~mm}^{3}$ small pieces, the cartilage was incubated with $0.2 \%$ collagenase II at $37^{\circ} \mathrm{C}$ for $3 \mathrm{~h}$. Finally, cell suspension was collected and cultured in DMEM/F12 (Gibco, USA) containing fetal bovine serum (Gibco, BRL) and $1 \%$ penicillin-streptomycin. Passage 1-3 from the primary cells were used for further experiments in this study.

\section{In vitro FSS Experiment}

FSS was applied by using a Flexcell FX-4000 strain unit (Flexcell, FX4000, Burlington, Ontario, Canada). FSS was 
calculated based on the following formula: $\tau=6 \mu \mathrm{Q} / \mathrm{wh}^{2}(\mu$ is the dynamic viscosity of perfusate, $\mathrm{Q}$ is the flow rate, $\mathrm{w}$ is the width, and $\mathrm{h}$ is the channel height). Briefly, chondrocytes were seeded onto the culture slips at a density of $2 \times 10^{5}$ cells and put into a parallel-plate flow system. The system is a six-chamber laminar flow device which includes a total of six $75 \times 25 \times 1 \mathrm{~mm}$ culture slips. After being exposed to 20 dyne $/ \mathrm{cm}^{2}$ FSS treatment for $2 \mathrm{~h}$, the cells were collected for further experiments.

\section{Cytoskeleton Staining}

The morphology of cells with or without shear stress treatment was investigated by phalloidin staining (Yeason, China). Briefly, cells were fixed in $4 \%$ paraformaldehyde for $30 \mathrm{~min}$ and then treated with $0.1 \%$ Triton X-100 solution for $10 \mathrm{~min}$. Cells were incubated with phalloidin staining for $30 \mathrm{~min}$ at room temperature. Finally, DAPI staining was carried out for $3 \mathrm{~min}$. Stained samples were observed by an inverted fluorescence microscope (Zeiss, Germany).

\section{Ethynyl-2'-Deoxyuridine (EdU) Staining}

Edu staining assay was utilized to evaluate cell viability. Briefly, cells were firstly incubated in $50 \mu \mathrm{M}$ EdU solution for $2 \mathrm{~h}$ at $37{ }^{\circ} \mathrm{C}$ incubator. Then, $4 \%$ paraformaldehyde was used to fix cells, followed by treatment with $2 \mathrm{mg} / \mathrm{mL}$ glycine and $0.5 \%$ Triton X-100. Afterwards, Apollo reaction solution was added into cells for reaction about 30 min in the dark. Finally, cell nuclei was stained by Hoechst 33342. Random visions were chosen for capturing images in different samples by using an inverted fluorescence microscope (Zeiss, Germany).

\section{RNA Isolation and RT-qPCR}

Chondrocytes were seeded at a density of $2 \times 10^{5}$ cells and subjected to different treatments. Total RNA of cells or tissues was extracted by TRIzol reagent (Takara, Japan) and then synthesized into cDNA by using a PrimeScript RT reagent kit (Takara, Japan). Real-time PCR was carried out on an ABI StepOne real-time PCR system (Applied Biosystems, USA) by using a SYBR Premix Ex Taq Reagent Kit (Takara, Japan). Reaction conditions were performed according to manufacturer's constructions. The relative expression level of genes was evaluated by the $2^{-\triangle \Delta C T}$ method with GAPDH used as the internal control. The detailed sequences of primers used in this study are presented in Table S1.

\section{Immunofluorescence Staining}

Firstly, samples were fixed by $4 \%$ paraformaldehyde and permeabilized by $0.1 \%$ Triton X-100. Then, samples were blocked by $10 \%$ goat serum plus $3 \%$ BSA after which immunostained with specific primary antibodies including COLII (1:50, proteintech, UK), SOX9 (1:50, Abcam, UK), COX-2 (1:50, CST, UK), MMP13 (1:50, proteintech, UK), $\mathrm{H} 3 \mathrm{~K} 4 \mathrm{me} 3$ (1:50, CST, UK) overnight at $4{ }^{\circ} \mathrm{C}$. After being reacted with fluorescent secondary antibody at room temperature for $1 \mathrm{~h}$, cells were stained with DAPI (Yeason, China) and observed under an inverted fluorescence microscope (Zeiss, Germany). Three different pictures were captured in random fields.

\section{Western Blot Assay}

Chondrocytes were seeded at a density of $2 \times 10^{5}$ cells and subjected to different treatments. Total protein of cells were extracted by SDS lysis buffer (Beyotime, China). Protein samples were subjected to $4-20 \%$ polyacrylamide gels (Genshare biological, China) electrophoresis and transferred to polyvinylidene fluoride (PVDF) membranes (Merck Millipore, USA). After being blocked with 5\% skim milk, membranes were incubated with specific primary antibodies against COLII (1:1000, proteintech, UK), SOX9 (1:1000, Abcam, UK), COX-2 (1:1000, CST, UK), MMP13 (1:1000, proteintech, UK), H3K4me3 (1:1000, CST, UK), H3K9me3 (1:1000, Abcam, UK), H3K27me3 (1:1000, CST, UK), H3K36me3 (1:1000, ABclonal, USA), H3K79me3 (1:1000, ABclonal, USA), KMT2B (1:1000, ABclonal, USA), $\beta$-catenin (1:1000, CST, UK), Histone H3 (1:2000, CST, UK), ZBTB20 (1:1000, proteintech, UK) and GAPDH $\left(1: 10,000\right.$, proteintech, USA) at $4{ }^{\circ} \mathrm{C}$ overnight. The membranes were then probed with secondary antibodies and visualized on an Amersham 600 Chemiluminescence System by using chemiluminescence (ECL) substrate kit (Merck Millipore, USA). All the Western blot bands were quantified by Image-Pro Plus 6.0 software and normalized to an internal control (GAPDH or Histone H3).

\section{RNA Sequencing and Differential Expression Analysis}

The transcriptome sequencing and differential expression analysis in this study were performed by OE Biotech Co., Ltd. (Shanghai, China). Briefly, constructed RNA libraries were sequenced on an Illumina HiSeq X Ten platform. DESeq (2012) R package was utilized to make differential 
expression analysis and differentially expressed genes (DEGs) in this study were defined as $\mathrm{P}$ value $<0.05$ and $|\log \mathrm{FC}|>1$.

RNA sequencing data was uploaded in Gene Expression Omnibus - NCBI database as GSE186501.

\section{CUT\&Tag Library Generation and Sequencing}

CUT\&Tag assay was performed using NovoNGS CUT\&Tag 3.0 High-Sensitivity Kit (for Illumina) (Novoprotein, China) as indicated. The detailed procedures were in accordance with previous protocols. ${ }^{20}$ Briefly, cells were treated with ConA beads after which were incubated with primary antibody for $2 \mathrm{~h}$ at room temperature. After being washed for three times, cells were reacted with secondary antibody for $1 \mathrm{~h}$. Subsequently, samples were incubated in pAG-Tn5 for $1 \mathrm{~h}$. DNA tagmentation was performed at $37^{\circ} \mathrm{C}$ for 1 $\mathrm{h}$ and the reaction was stopped with $10 \% \mathrm{SDS}$ at $55^{\circ} \mathrm{C}$ for $10 \mathrm{~min}$. After extraction with phenol-chloroform and ethanol precipitation, PCR was applied to amplify the libraries. Sequencing and data analysis was performed by GENEWIZ (Suzhou, China).

\section{Adenovirus Overexpression of ZBTB20}

GFP-ZBTB20 adenoviral vectors were constructed by HanBio Technology (Shanghai, China). The multiplicity of infection was 400 . When primary chondrocytes reached the confluence of about $30-50 \%$, cells were infected with adenovirus in DMEM/F12 for $8 \mathrm{~h}$ at $37{ }^{\circ} \mathrm{C}$.

\section{Flow Cytometry}

The apoptosis activities of chondrocytes were determined by flow cytometry analysis.

Primary chondrocytes were seeded onto 6-well plate at a density of $2 \times 10^{5}$ cells and then exposed to different treatments. After being washed by cold PBS for three times, samples were stained with Annexin V-APC/PI Apoptosis Detection Kit (KeyGEN BioTECH, China) to assess apoptosis activities. Specifically, cell suspension was stained with $5 \mu \mathrm{L}$ PI staining buffer and $5 \mu \mathrm{L}$ Annexin $\mathrm{V}$ staining buffer protected from light for about $15 \mathrm{~min}$ and then analyzed on a Flow Cytometer (BD Biosciences, USA).

\section{In vitro MM-I02 or LF3 Treatment}

MM-102 (Sellect, USA) and LF3 (Sellect, USA) were dissolved in DMSO at a storage concentration at $-40{ }^{\circ} \mathrm{C}$ until use. A density of $2 \times 10^{5}$ chondrocytes were seeded at 6-well plate and pretreated with $20 \mu \mathrm{M}$ MM-102 or 20 $\mu \mathrm{M}$ LF3 for $24 \mathrm{~h}$ after which subjected to shear stress or $500 \mu \mathrm{M}$ sodium nitroprusside (SNP) treatment. SNP was commonly used to mimic apoptosis of chondrocytes.

\section{Unilateral Anterior Crossbite (UAC) Model}

A total of 22 male C57BL/6J mice (19-22g, 6-week-old) were provided by the Shanghai Laboratory Animal Center and all the experimental procedures were conducted according to the Guide for Care and Use of Laboratory Animals (Ministry of Science and Technology of China, 2006). All the mice were randomly divided into UAC group or sham group. The UAC animal model used in this study for inducing temporomandibular joint arthritis (TMJOA) was described previously. ${ }^{21,22}$ Briefly, metal tubes were used to bond left maxillary and mandibular incisors to create UAC relationship. The mandibular tubes were curved to form a $135^{\circ}$ labially inclined occlusal plate. Mice in the sham groups underwent similar procedures without bonded metal tubes.

\section{Micro-CT Analysis}

Temporomandibular joint (TMJ) tissues of C57BL/6J mice in different group were obtained and fixed in $4 \%$ paraformaldehyde. The slice thickness for the micro-CT scans was $9.13 \mu \mathrm{m}$ and a total of 450 slices of bone were scanned for Micro-CT analysis on skyscan1076 (Brooke, Belgium). Specifically, 2 cubes (each $0.3 \times 0.3 \times 0.3 \mathrm{~mm}$ ) were selected from the midcenter and midposterior of condylar subchondral bone to be analyzed by CTvol (greylevel threshold 120-255) for the parameters including the ratio of bone volume to tissue volume (BV/TV), bone mineral density (BMD), trabecular thickness ( $\mathrm{Tb} . \mathrm{Th}$ ), trabecular number (Tb. N) and trabecular space ( $\mathrm{Tb} . \mathrm{Sp})$.

\section{Histology Analysis and Immunohistochemistry Assay}

TMJ tissues were collected and demineralized in $10 \%$ ethylenediaminetetraacetic acid (EDTA) solution. Afterwards, tissue samples were embedded in paraffin and sectioned at a thickness of approximately $5 \mu \mathrm{m}$. Samples were deparaffinized, hydrated, and stained with hematoxylin-eosin (H\&E) and Safranin-O-fast green. We adopted OARSI Score criteria to evaluate cartilage degeneration as previously stated. ${ }^{23}$ For immunohistochemistry assay, after being deparaffinized and 
rehydrated, samples were immersed in $0.3 \%$ hydrogen peroxide to block endogenous peroxidase activities. Subsequently, samples were incubated with proteinase $\mathrm{K}$ for antigen retrieval after which blocked with $1 \%$ goat serum plus 3\% BSA solution. Primary antibodies against COLII (1:50, Proteintech, USA), SOX9 (1:200, CST, UK), COX-2 (1:50, CST, UK), MMP13 (1:50, Proteintech, USA), H3K4me3 (1:50, CST, UK), and ZBTB20 (1:50, Proteintech, USA) were used to incubate specimen at $4{ }^{\circ} \mathrm{C}$ overnight. After being reacted with the secondary antibody for $1 \mathrm{~h}$ at room temperature, samples were stained with hematoxylin, dehydrated, cleared and mounted. Images were analyzed by Image-Pro Plus 6.0 software and the percentage of positive cells was presented.

\section{Statistical Analysis}

All the experiments were performed for three times. Statistical analysis was performed by SPSS 19.0 software. Comparisons between two groups or three groups were evaluated by Student's $t$ tests and one-way analysis of variance (ANOVA), respectively. $\mathrm{P}<0.05$ was determined as statistically significant throughout this study.

\section{Results}

\section{FSS Influences the Morphology and Viability of Primary Chondrocytes}

In this study, we used Flexcell appliance to exert FSS on chondrocytes and the detailed schematic diagram was presented in Figure S1. Briefly, cells were seeded in the culture slips $48 \mathrm{~h}$ before FSS treatment. To detect the biological effect of aberrant high shear stress on chondrocytes, we applied $2 \mathrm{~h}$ treatment of 20 dyne $/ \mathrm{cm}^{2}$ FSS. Cytoskeleton staining showed that FSS significantly disrupt cell morphology, mainly manifested by the irregular display of F-actin (Figure 1A). Furthermore, EdU staining which could label cells in S-phase indicated the decreased cell proliferation and viability of primary chondrocytes under FSS (Figure 1B).

\section{FSS Induces Proinflammatory Mediators and Leads to Cartilage Degeneration and Degradation}

We further investigated the effect of FSS on cartilagerelated markers, catabolism responses, inflammatoryrelated factors, and extracellular matrix degradation. It is shown in Figure 1C that the mRNA level of Sox9, Acan, and Col2 mRNA was remarkably downregulated after FSS treatment. Meanwhile, hypertrophy-related markers, such as Col10, Cox-2, Adamts4, and Adamts5 were significantly upregulated. Multiple matrix metalloproteinases, including Mmp3, Mmp9, and Mmp13, and typical inflammatory factors (TNF- $\alpha$, IL-1 $\beta$, and IL-6) were also induced. Western blot results demonstrated the suppression of COLII and SOX9 and a remarkable elevated level of COX-2 and MMP13 after applying FSS (Figures 1D and S2). Immunofluorescence assay consistently showed the negative regulatory roles of aberrant FSS in primary chondrocytes (Figure 1E).

\section{FSS Results in $\mathrm{H} 3 \mathrm{~K} 4 \mathrm{me} 3$ Activation and KMT2B Upregulation}

Since histone methylation is one of the most significant epigenetic regulation for gene expression, ${ }^{24}$ we chose to detect major histone methylation modifications (H3K4me3, H3K9me3, H3K27me3, H3K36me3, and H3K79me3) in FSS-treated chondrocytes. The results suggested that FSS leads to a remarkable overexpression of $\mathrm{H} 3 \mathrm{~K} 4 \mathrm{me} 3$ and a silence of $\mathrm{H} 3 \mathrm{~K} 27 \mathrm{me} 3$ (Figures 2A and $\underline{\mathrm{S} 3}$ ). Since $\mathrm{H} 3 \mathrm{~K} 4 \mathrm{me} 3$ is defined as a major epigenetic controller which was closely associated with gene activation, we chose to further investigate the underlying mechanism of $\mathrm{H} 3 \mathrm{~K} 4 \mathrm{me} 3$ in the responses of chondrocytes to FSS. Consistently, immunofluorescence assay indicated a remarkable upregulation of $\mathrm{H} 3 \mathrm{~K} 4 \mathrm{me} 3$ in chondrocytes exposed to FSS (Figure 2B). Subsequently, we determined to investigate the potential mechanism underlying FSSinduced $\mathrm{H} 3 \mathrm{~K} 4 \mathrm{me} 3$ activation. Analysis of histone methyltransferases revealed that the mRNA expression of $\mathrm{Kmt} 2 \mathrm{~b}$ was remarkably elevated after FSS treatment (Figure 2C). Then, Western blot and semi-quantitative analysis suggested an obvious upregulation of KMT2B protein level in chondrocytes under FSS, implying that $\mathrm{H} 3 \mathrm{~K} 4 \mathrm{me} 3$ activation may result from KMT2B upregulation $(\mathrm{P}<0.01$, Figure $2 \mathrm{D})$.

\section{Pharmacological Inhibition of H3K4me3 Activation by MM-I02 Partially Rescues the Destructive Effect of FSS on \\ Chondrocytes}

Given the important mediatory effect of H3K4me3 activation on $\mathrm{OA}$, we intended to investigate whether its inhibition by MM-102 has therapy prospect. SNP was commonly used to mimic apoptosis of chondrocytes. Firstly, we pretreated cells with $20 \mu \mathrm{M}$ MM-102 for $24 \mathrm{~h}$ and then incubated with $500 \mu \mathrm{M}$ SNP. Apoptosis 
A

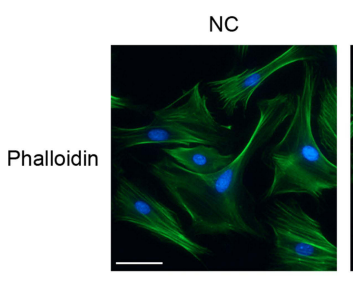

C
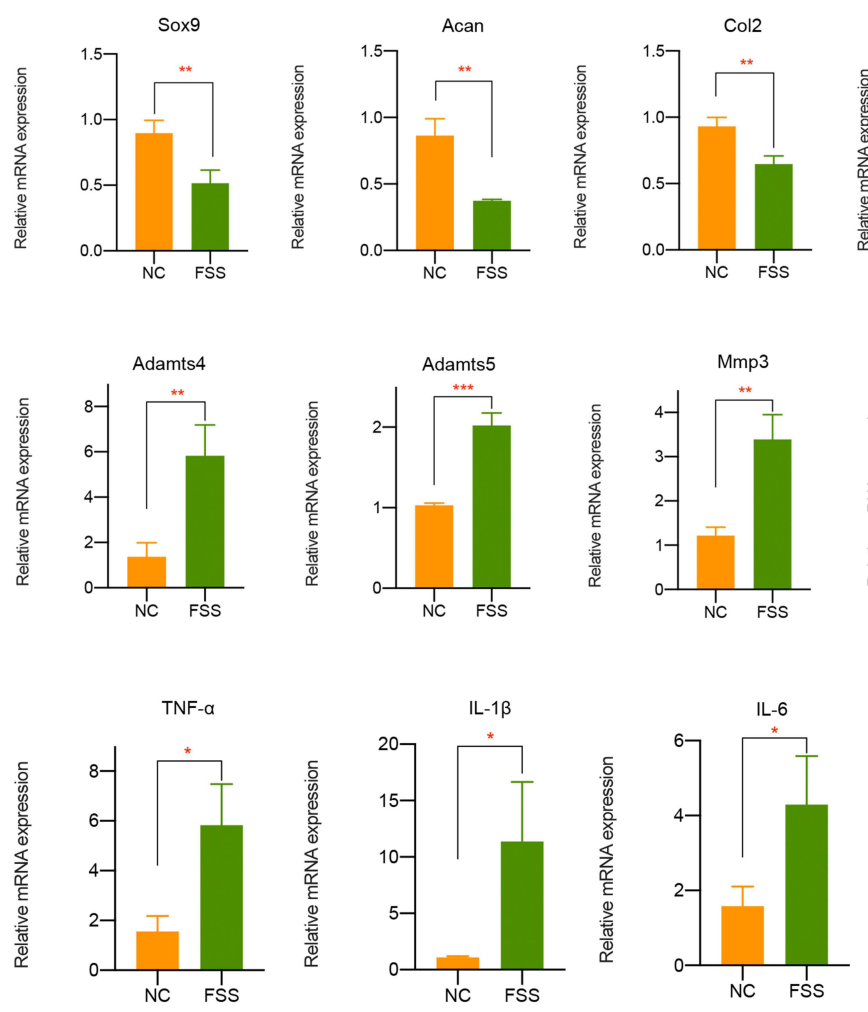
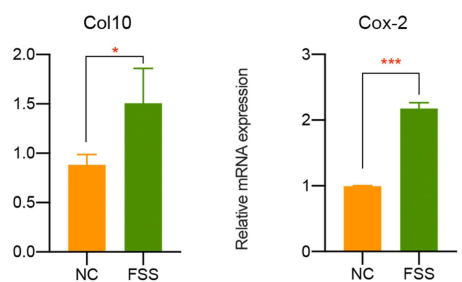

\section{E}
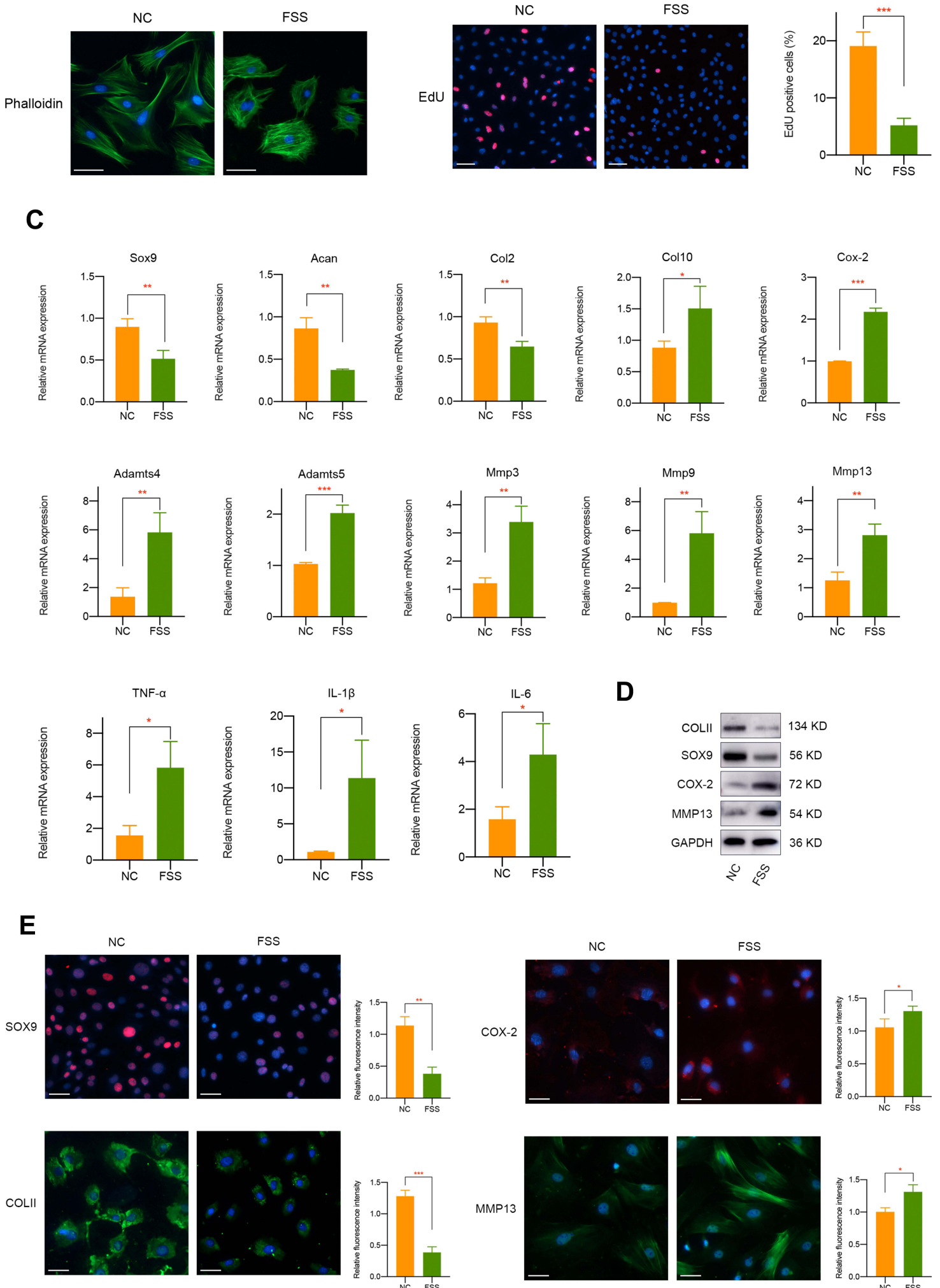

Figure I The biological effect of aberrant FSS on primary chondrocytes. (A) FSS affects the morphology of primary chondrocytes. (B) FSS significantly decreases primary chondrocytes viability. (C) mRNA level of cartilage-related markers, hypertrophy-related markers and proinflammatory mediators was regulated by FSS. (D) Western blot assay demonstrated the protein expression of COLII, SOX9, COX-2, and MMPI3 was mediated by FSS. (E) Immunofluorescence assay indicated the protein expression of COLII, SOX9, COX-2, and MMPI3 was mediated by FSS. Scar bar, $50 \mu \mathrm{m}$. $*_{\mathrm{p}}<0.05$, **p $<0.01$, *** $\mathrm{p}<0.001$. 
A

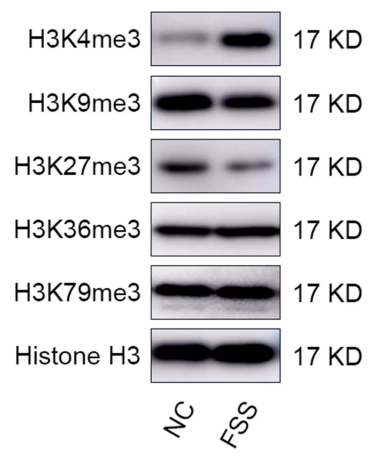

B

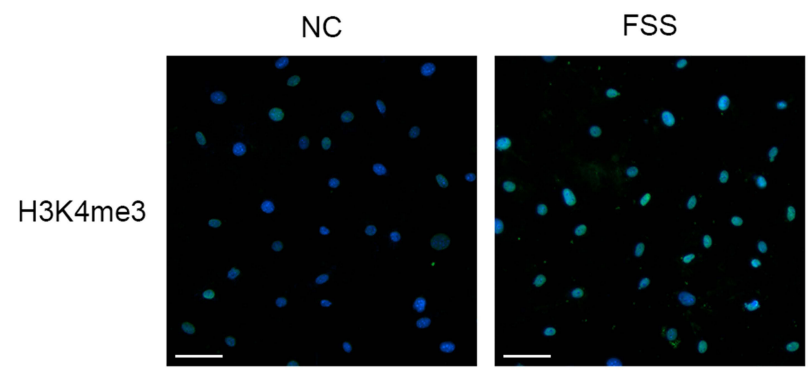

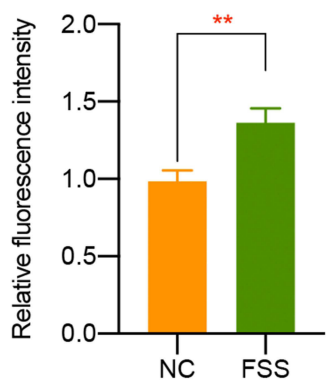

C
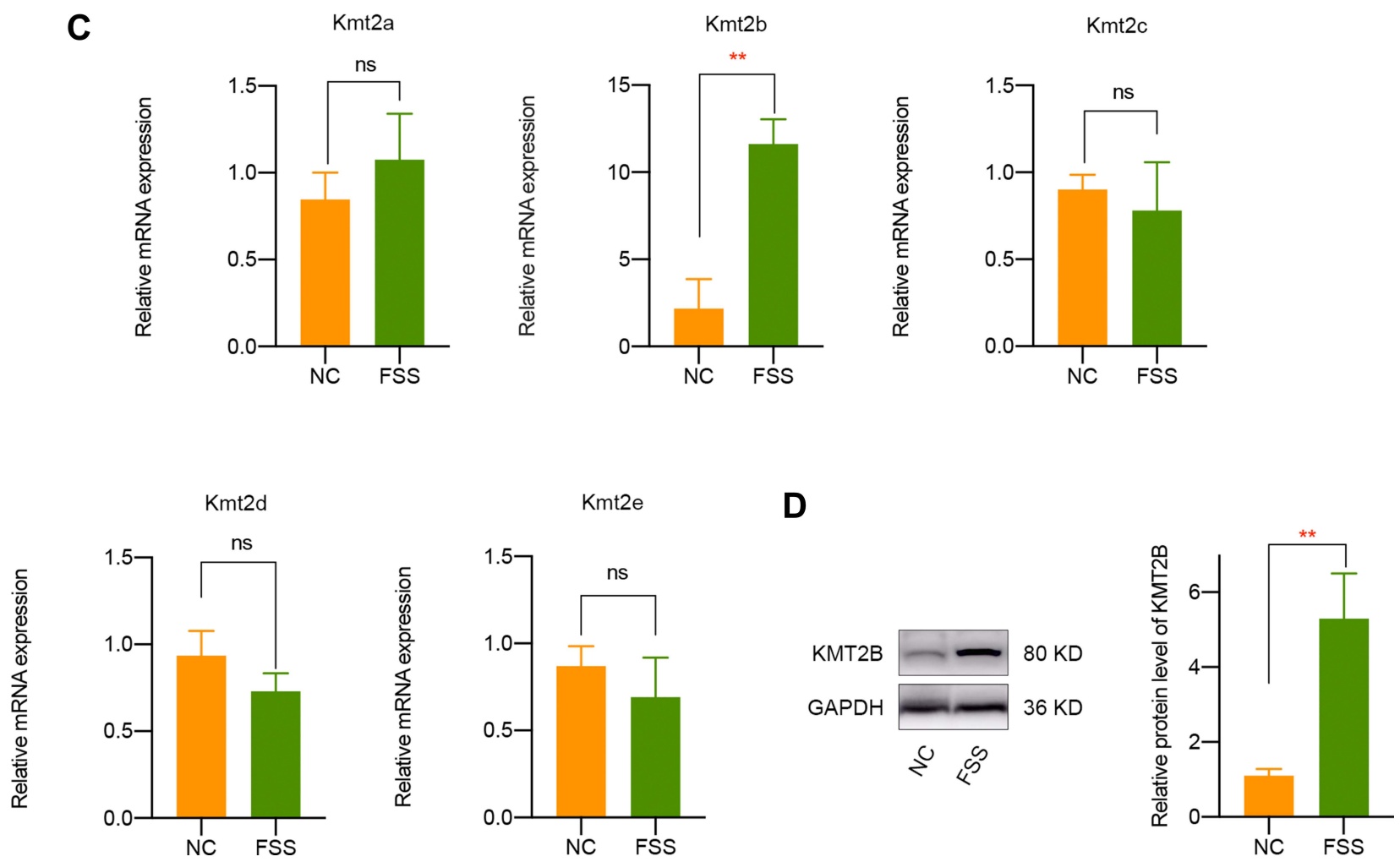

Figure 2 FSS results in $\mathrm{H} 3 \mathrm{~K} 4 \mathrm{me} 3$ activation and $\mathrm{KMT} 2 \mathrm{~B}$ upregulation. (A) Western blot results of $\mathrm{H} 3 \mathrm{~K} 4 \mathrm{me} 3, \mathrm{H} 3 \mathrm{~K} 9 \mathrm{me}, \mathrm{H} 3 \mathrm{~K} 27 \mathrm{me} 3, \mathrm{H} 3 \mathrm{~K} 36 \mathrm{me}$, and $\mathrm{H} 3 \mathrm{~K} 79 \mathrm{me} 3$ expression in chondrocytes exposed to FSS. (B) Immunofluorescent staining of H3K4me3 in chondrocytes after FSS treatment. (C) The mRNA level of H3K4 methyltransferases in chondrocytes under static or FSS conditions. (D) The protein expression of KMT2B was significantly upregulated after FSS treatment. Scar bar, 50 $\mu \mathrm{m} .{ }^{* *} \mathrm{p}<0.01$, ns, not significant.

activities were evaluated about $24 \mathrm{~h}$ later and it was shown that SNP significantly stimulated chondrocytes apoptosis while cells pretreated with MM-102 alleviate the destructive effect of SNP (Figure 3A). More importantly, MM-102 pretreatment effectively rescued the negative impact of FSS on chondrocytes, which may lay a foundation of epigenetic-based therapy on OA (Figure 3B and $\mathrm{C}$ ).
Identification of ZBTB20 as H3K4me3-Target Gene Mediated by FSS

To make an in depth investigation of the molecular mechanism during FSS-induced OA, we firstly analyzed the transcriptomic effects of FSS on chondrocytes. A total of 430 genes were determined as upregulated by the criteria of $p<0.05$ and fold change $>2$. CUT\&Tag sequencing was utilized to confirm the direct target genes of 
$\mathrm{H} 3 \mathrm{~K} 4 \mathrm{me} 3$. Analysis results indicated that 586 genes were regulated by $\mathrm{H} 3 \mathrm{~K} 4 \mathrm{me} 3$. Through the intersection of RNAseq of chondrocytes exposed to FSS and CUT\&Tag sequence of $\mathrm{H} 3 \mathrm{~K} 4 \mathrm{me} 3$ in cells with or without FSS treatment, 10 genes (Capn12, Cldn1, Gpcpd1, I113ra2, Mex3b, Nexn, Slc20a1, Srek1, Tgif1, and Zbtb20) were finally screened out (Figure 4A). Further qPCR validation indicated that $\mathrm{Zbtb} 20$ is the most significant upregulated gene after FSS treatment (Figure 4B). Finally, we selected ZBTB20 as promising $\mathrm{H} 3 \mathrm{~K} 4 \mathrm{me} 3$-target gene and the peak of ZBTB20 in corresponding group is displayed in Figure 4C. CUT\&Tag verification suggested Zbtb20 was directly targeted by $\mathrm{H} 3 \mathrm{~K} 4 \mathrm{me} 3$ activation caused by FSS (Figure 4D). Moreover, ZBTB20 protein expression was also significantly elevated after FSS $(\mathrm{P}<0.001$, Figure $4 \mathrm{E}$ ). Both the elevated mRNA level and protein level of ZBTB20 was rescued by MM-102 pretreatment under FSS (Figure 4F and G). The above results demonstrated that ZBTB20 was directly regulated by FSSinduced $\mathrm{H} 3 \mathrm{~K} 4 \mathrm{me} 3$ activation.

\section{ZBTB20 Regulates the Biological Phenotypes of Chondrocytes Through Targeting Wnt Signaling}

To detect the modulatory effect of ZBTB20 on chondrocytes, cells were infected with adenovirus for ZBTB20 overexpression. qPCR and Western blot results validated the success overexpression of ZBTB20 in primary chondrocytes (Figure 5A). It is shown in Figure 5B that apoptosis activities were induced in Ad-ZBTB20-treated chondrocytes. Moreover, ZBTB20 was identified to suppress the mRNA expression of Col2, Sox9, and Acan, implying its negative impact on chondrocytes (Figure 5C). Hypertrophy and catabolism-related markers, including Col10, Cox-2, Adamts4, and Adamts5 were remarkably upregulated (Figure 5C). Matrix metalloproteinases (Mmp3, Mmp9, and Mmp13) and inflammatory factors (TNF- $\alpha$, IL-1 $\beta$, and IL-6) were also induced, suggesting matrix degradation and inflammatory responses induced by ZBTB20 (Figure 5C). Consistently, the protein expression of COLII and SOX9 were significantly downregulated and a statistical significant elevated level of COX-2 and MMP13 was observed after ZBTB20 overexpression (Figure 5D). Since Wnt signaling is an important biological cascade which is mechanical responsive and closely involved in joint homeostasis and joint diseases ${ }^{25,26}$ and the KEGG enrichment analysis of chondrocytes with or without FSS contains the Wnt pathway (Figure S4), we determined to detect whether ZBTB20 exerts its biological functions through mediating Wnt pathway. Western blot results showed that ZBTB20 overexpression leads to Wnt signaling activation, with a more than 3 -fold upregulation of $\beta$-catenin level $(\mathrm{P}<0.05$, Figure $6 \mathrm{~A})$. Further rescue experiments suggested that treatment with $\mathrm{Wnt} / \beta$-catenin inhibitor LF3 could effectively alleviate the negative effect of ZBTB20 on chondrocytes, mainly manifested by decreased cell apoptosis (Figure 6B). Also, cartilagerelated positive markers Sox9, Col2, and Acan were improved and markers representing cartilage degeneration and inflammatory responses were decreased in LF3-treated chondrocytes with ZBTB20 overexpression (Figures 6C and $\underline{\text { 5 A }) . ~ W e s t e r n ~ b l o t ~ e x p e r i m e n t ~ s h o w e d ~ t h e ~ s a m e ~}$ results, manifested by the upregulation of COLII and SOX9 and downregulation of COX-2 and MMP13 in LF3treated cells (Figures 6D and $\underline{\mathrm{S} 6}$ ).

\section{Wnt Signaling Pathway is Involved in FSS-Related Cartilage Pathogenesis}

Firstly, we found that FSS leads to Wnt signaling activation (Figure 6E). To further detect whether Wnt signaling pathway was involved in aberrant FSS-induced cartilage pathogenesis, specific Wnt/ $\beta$-catenin inhibitor LF3 was used for rescue experiments. It was indicated that pretreated with LF3 could rescue the apoptosis activities caused by SNP treatment (Figure 6F). Furthermore, Wnt/ $\beta$-catenin inhibition by LF3 could alleviate the destructive effect of FSS on primary chondrocytes, represented by elevated level of COLII and SOX9 and decreased level of COX-2 and MMP13 (Figures 6G and H, S5B and S7). Therefore, we speculated that FSS destroys cartilage through KMT2BH3K4me3-ZBTB20-mediated Wnt signaling activation.

\section{Validation of $\mathrm{H} 3 \mathrm{~K} 4 \mathrm{me} 3$ and ZBTB20 in UAC Mice Model}

In vivo, we adopted UAC model which could produce abnormalities in bite force direction and distribution to mimic the natural pathogenesis process of aberrant occlusion-induced TMJOA in human beings as previously described. $^{21,27,28}$ Micro-CT analysis results suggested a decrease of BMD, BV/TV, Tb.Th, and Tb.N in UAC group while Tb.Sp was increased when compared to sham controls (Figure 7A). mRNA expression level of Col2 and Sox9 was downregulated, Cox-2 and Mmp13 was upregulated in UAC group (Figure 7B). H\&E staining and 

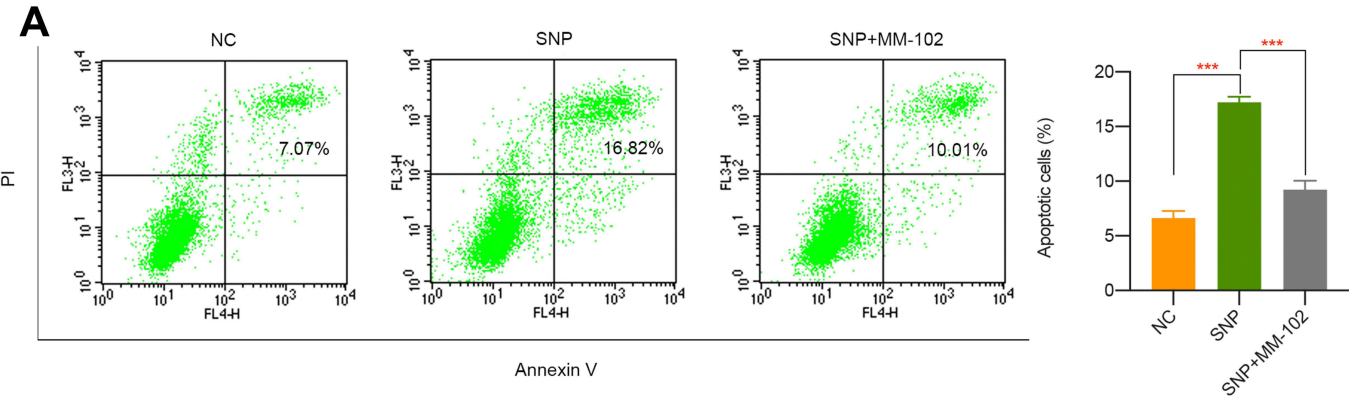

\section{B}
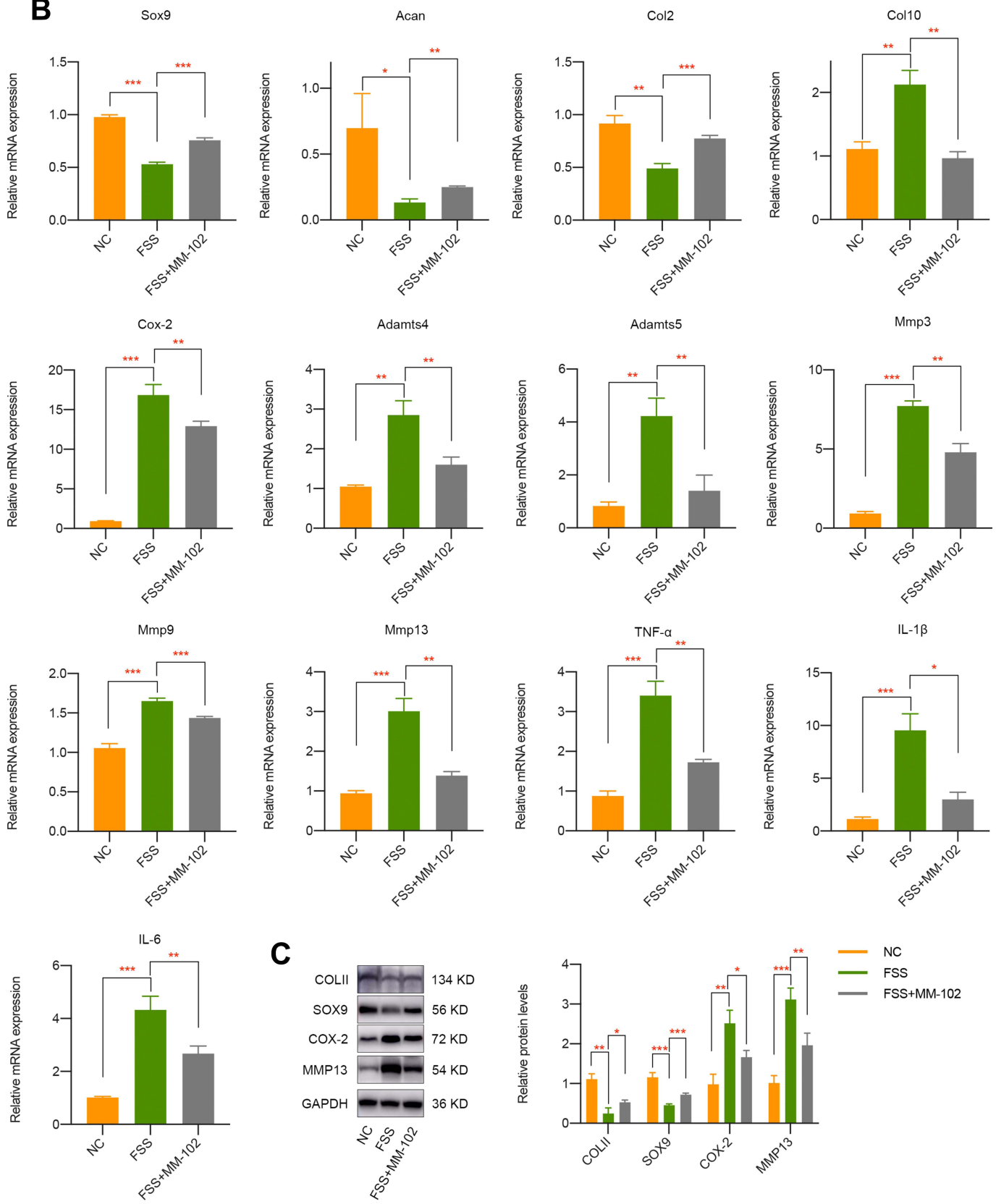

Figure 3 Pharmacological inhibition of H3K4me3 activation by MM-102 partially rescues the negative effect of FSS on chondrocytes. (A) Pretreatment of MM-102 in primary chondrocytes could alleviate SNP-induced apoptosis activities. (B) qPCR results indicated the inhibition of H3K4me3 activation by MM- 102 partially rescues the negative effect of FSS on chondrocytes. (C) Western blot results of COLII, SOX9, COX-2, and MMPI3 in chondrocytes exposed to FSS with or without MM-102 pretreatment. ${ }^{*} p<0.05,{ }^{* *} p<0.01$, ***p $<0.001$. 
A

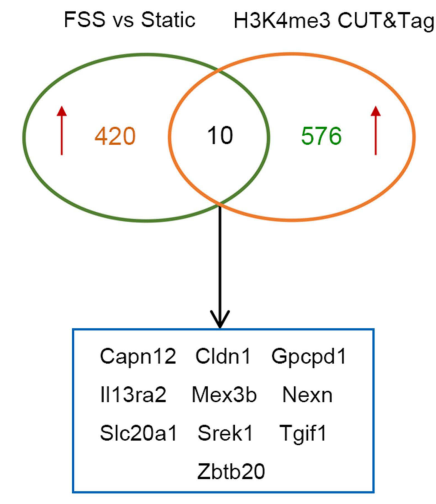

B

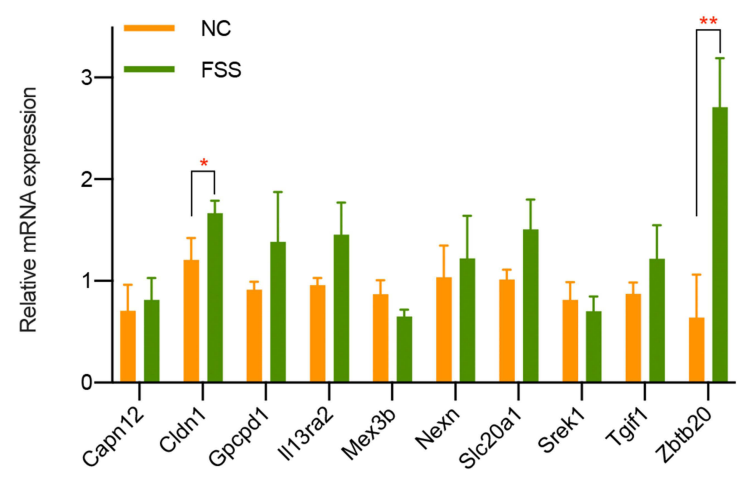

\section{C}

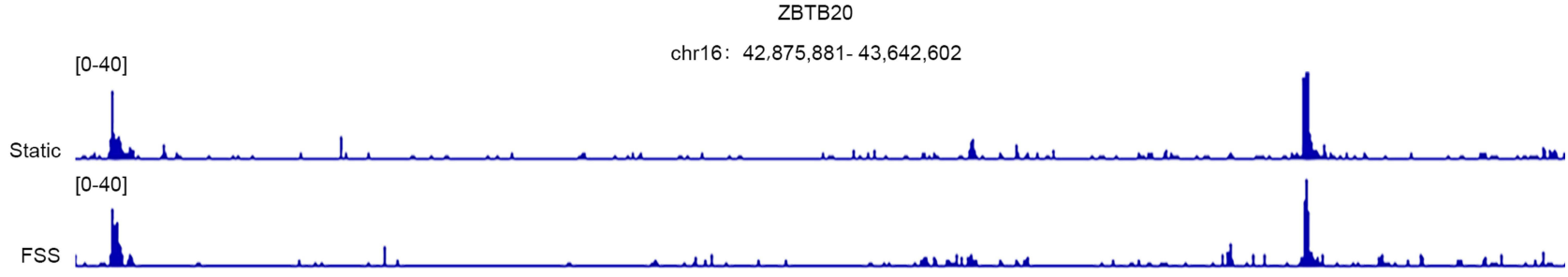

D

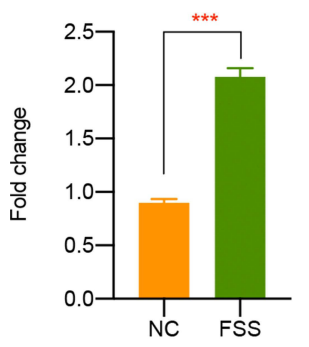

$\mathbf{E}$
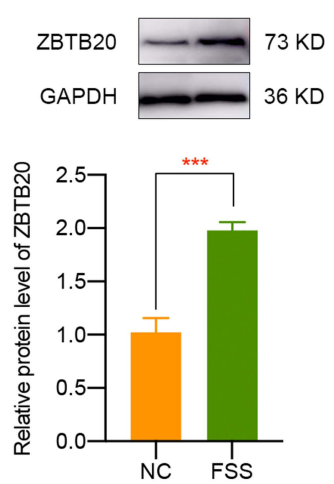

$\mathbf{F}$

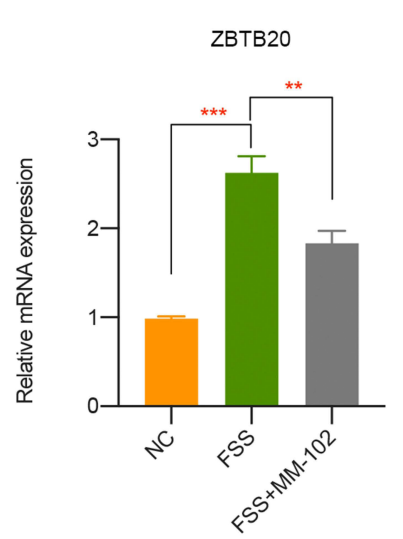

G

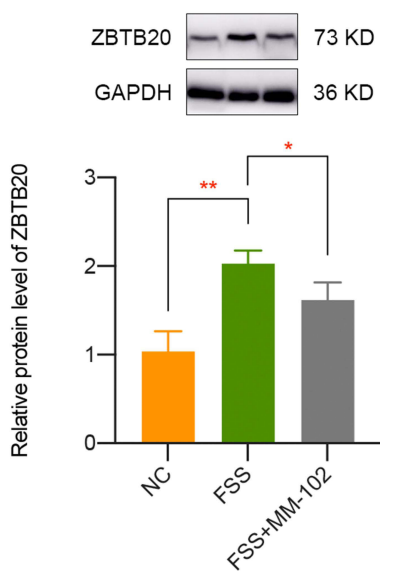

Figure 4 Identification of ZBTB20 as H3K4me3-target gene in chondrocytes exposed to FSS. (A) Overlapping differentially expressed genes was identified by the intersection of RNA-seq of chondrocytes exposed to FSS and CUT\&Tag sequence of H3K4me3 in cells with or without shear stress treatment. (B) qPCR validation of candidate H3K4me3-target genes caused by FSS. (C) Representative IGV overview of CUT\&Tag signals of H3K4me3 in the promoter regions of ZBTB20. (D) CUT \& Tag validation showed increased occupancy of ZBTB20 promoter in chondrocytes exposed to FSS. Data are shown as fold enrichments to the lgG control. (E) The protein level of ZBTB20 is significantly induced by FSS. (F) Inhibition of H3K4me3 by MM-I02 could suppress the elevated mRNA level of Zbtb20 caused by FSS. (G) Pretreatment with MM-I 02 inhibited the upregulated protein level of ZBTB20 by FSS. ${ }^{*} p<0.05$, **p $<0.01$, ***p $<0.001$.

Safranin O staining showed cartilage degradation and degeneration in UAC mice, which was illustrated by an elevated OARSI score and a decreased chondrocyte density $(\mathrm{P}<0.001$, Figure $7 \mathrm{C})$. Immunohistochemistry assay results indicated that tissues from UAC group presented a decreased positive staining intensity of COLII and SOX9 and an increased protein expression of COX-2 and MMP13 could be observed in UAC mice compared to sham controls $(\mathrm{P}<0.001$, Figure 7D). These findings implies cartilage degradation and degeneration in UAC 
A
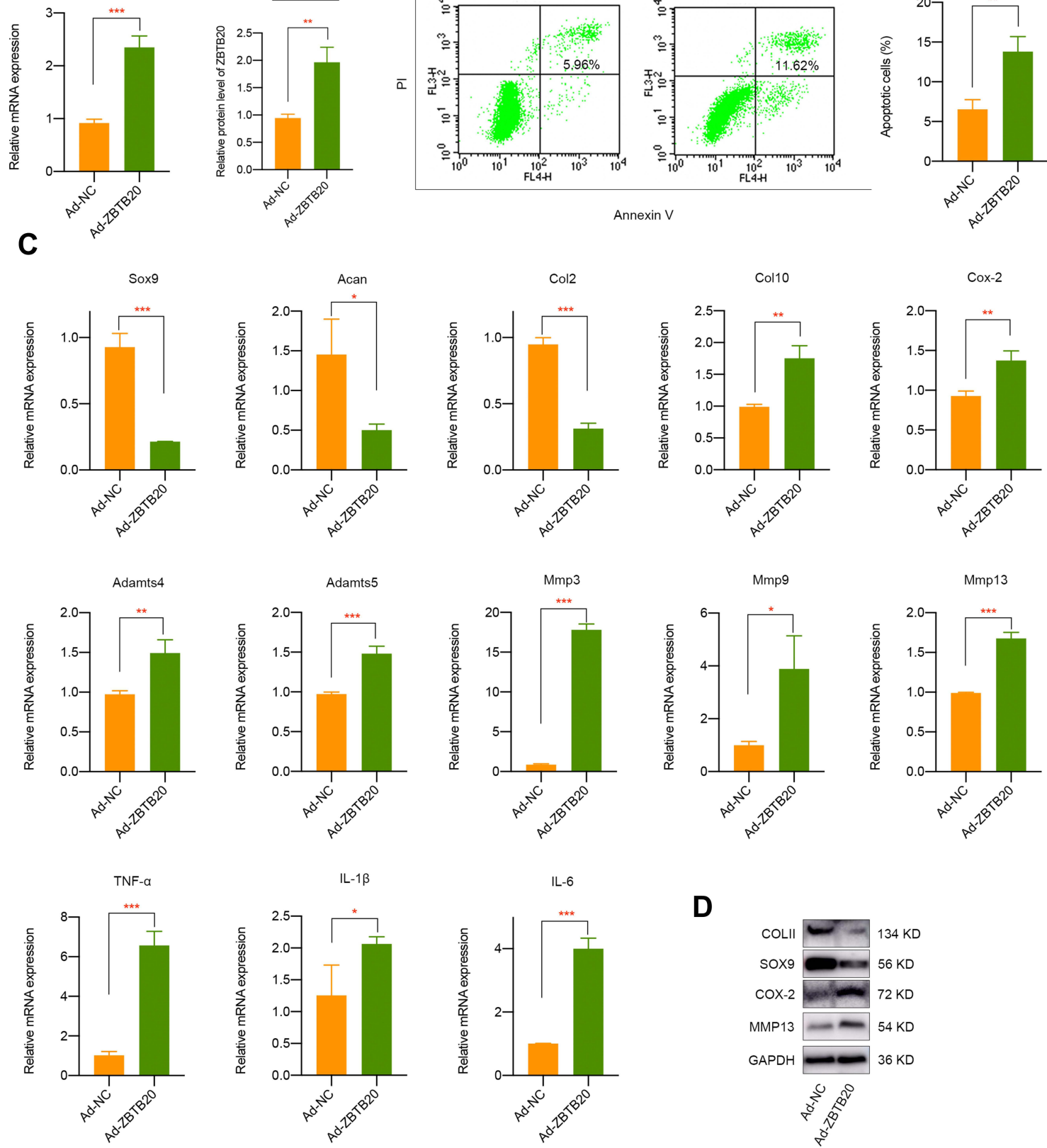

D

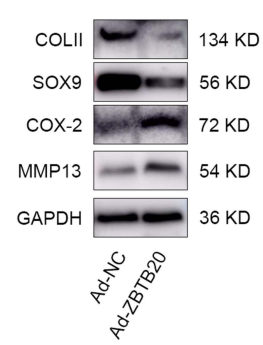

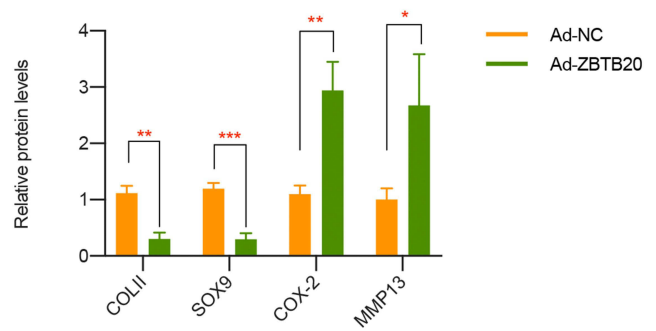

Figure 5 ZBTB20 remarkably affects the biological phenotypes, cartilage degeneration, and inflammatory responses in primary chondrocytes. (A) ZBTB20 was significantly elevated in primary chondrocytes with Ad-ZBTB20 infection at both mRNA and protein level. (B) Overexpression of ZBTB20 in chondrocytes greatly induces apoptosis activities. (C) qPCR results of markers representing cartilage degeneration, catabolic and inflammatory responses in chondrocytes with ZBTB20 overexpression. (D) The protein expression of COLII, SOX9, COX-2, and MMPI3 in chondrocytes was mediated by ZBTB20. ${ }_{p} p<0.05$, **p $<0.01$, ***p $<0.001$. 
A
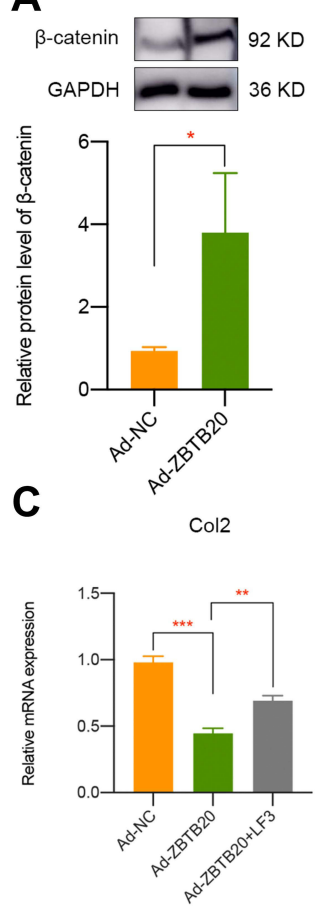

E

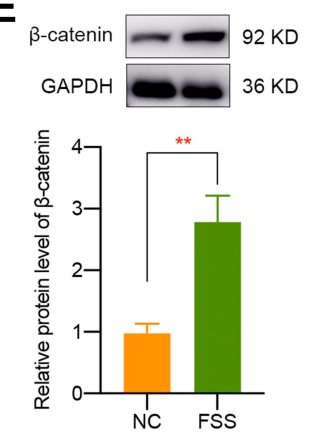

G

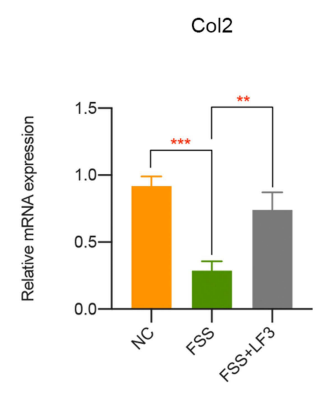

B
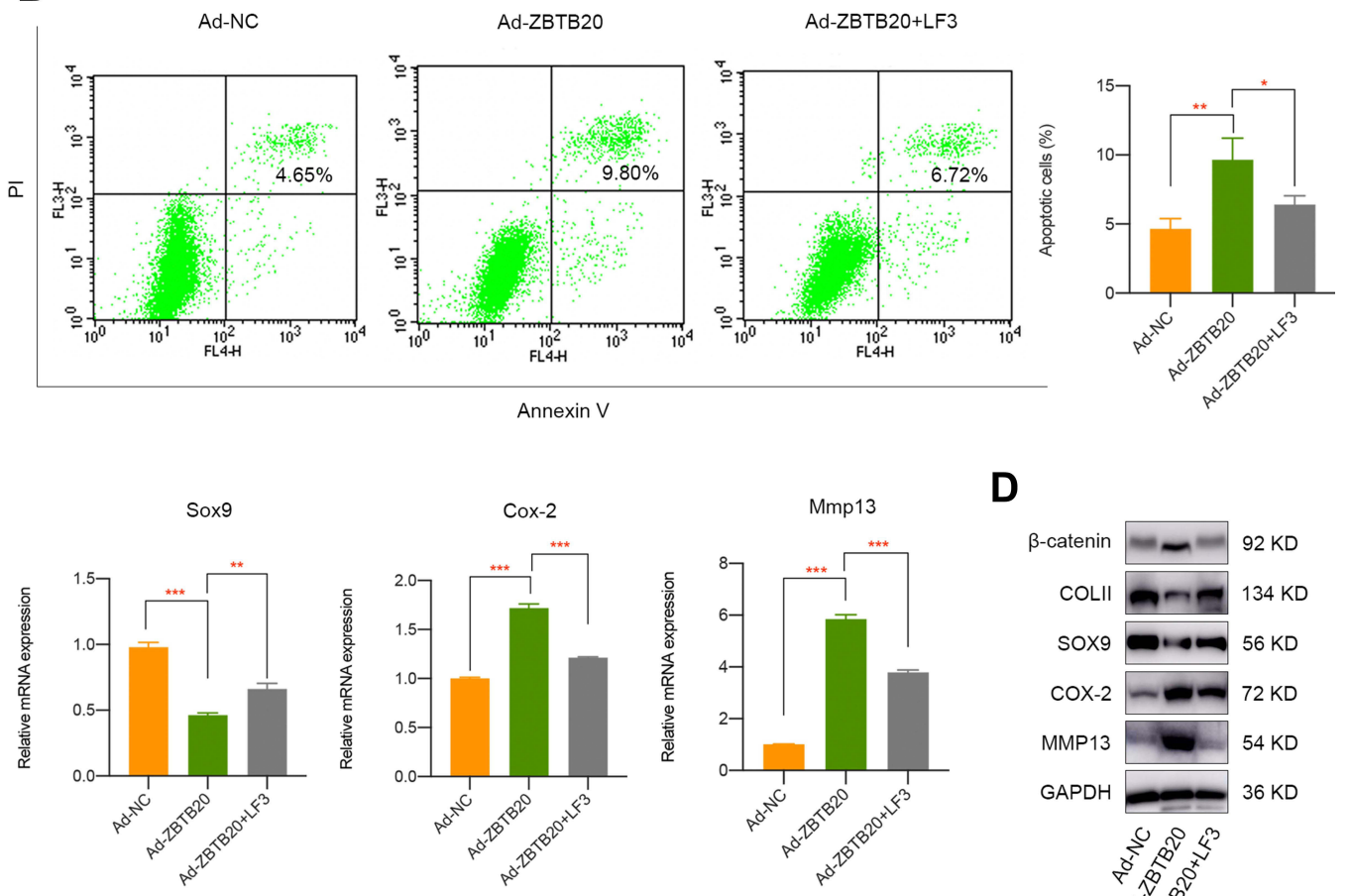

D
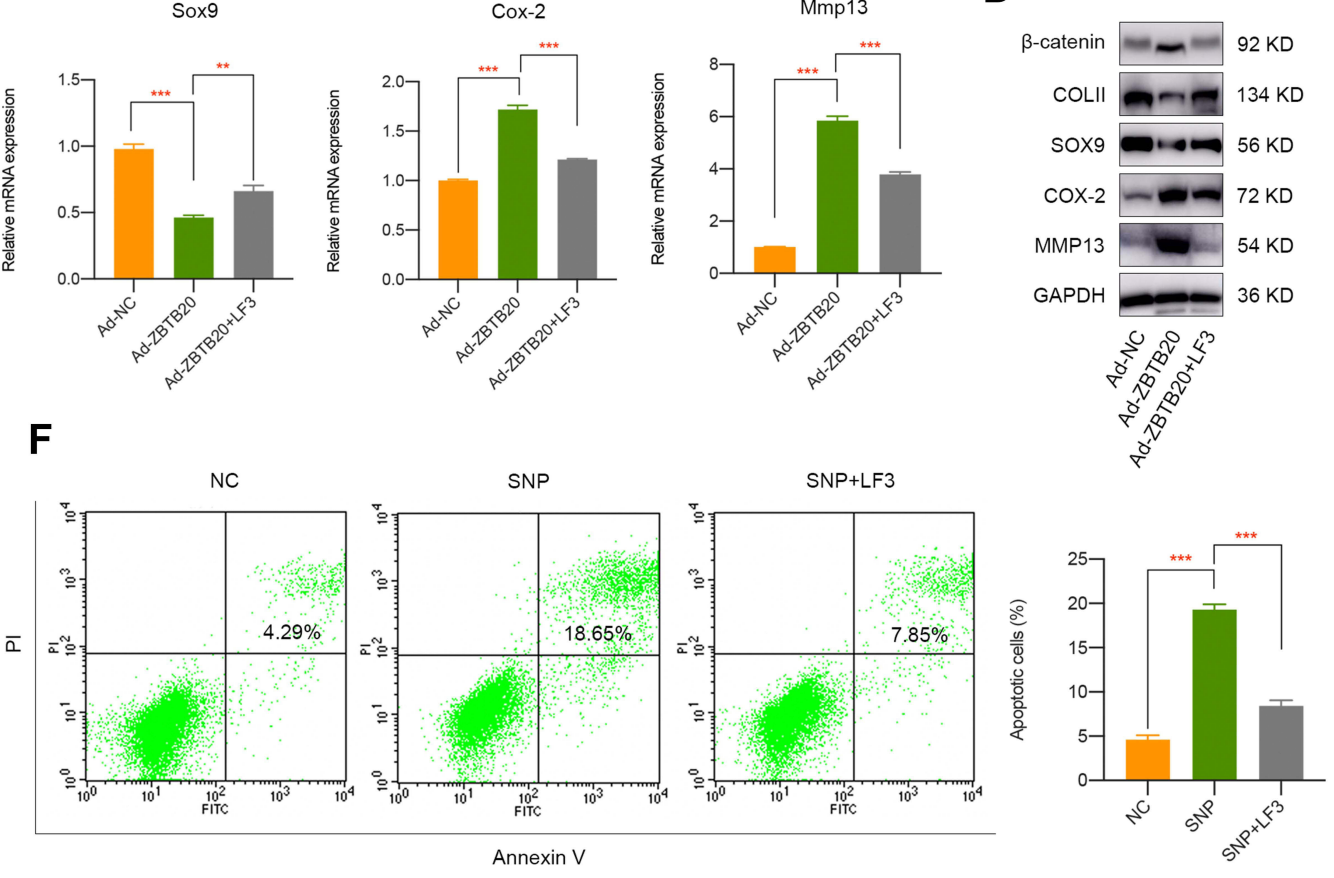

H
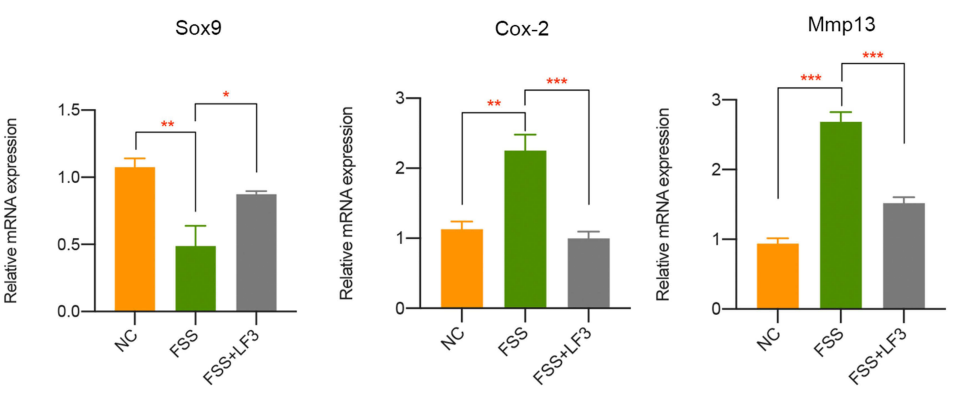

Figure 6 Wnt signaling pathway is closely involved in ZBTB20 or FSS-mediated cartilage pathogenesis. (A) Overexpression of ZBTB20 in primary chondrocytes results in Wnt signaling activation. (B) Treatment with LF3 decreased cell apoptosis induced by ZBTB20. (C) qPCR results indicated the inhibition of Wnt signaling by LF3 partially rescues the negative effect of ZBTB20 on chondrocytes. (D) Western blot results of COLII, SOX9, COX-2, and MMPI3 in Ad-NC or Ad-ZBTB20 infected chondrocytes with or without LF3 treatment. (E) FSS results in Wnt signaling activation. (F) Pretreatment of LF3 in chondrocytes could alleviate SNP-induced apoptosis activities. (G) qPCR results indicated the inhibition of Wnt signaling by LF3 partially rescues the negative effect of FSS on chondrocytes. (H) Western blot results of COLII, SOX9, COX-2, and MMPI 3 in chondrocytes exposed to FSS with or without LF3 treatment. ${ }^{*} p<0.05$, ${ }^{* *} p<0.01$, ***p $<0.001$.

mice. Furthermore, a strong activation of $\mathrm{H} 3 \mathrm{~K} 4 \mathrm{me} 3$ and ZBTB20 was observed in the TMJ tissues of UAC mice, as manifested by a great larger number of $\mathrm{H} 3 \mathrm{~K} 4 \mathrm{me} 3$ or
ZBTB20-positive cells $(\mathrm{P}<0.001$, Figure 7D). The dysregulation of H3K4me3 and ZBTB20 in aberrant mechanical force-induced TMJOA model implied the potential 
A

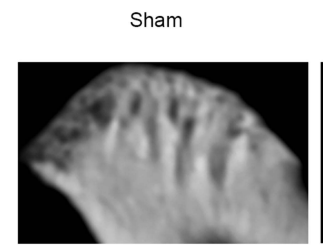

UAC

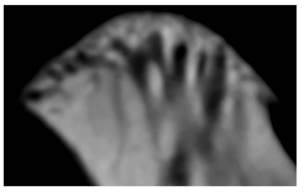

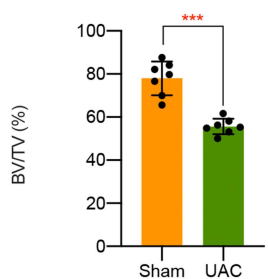
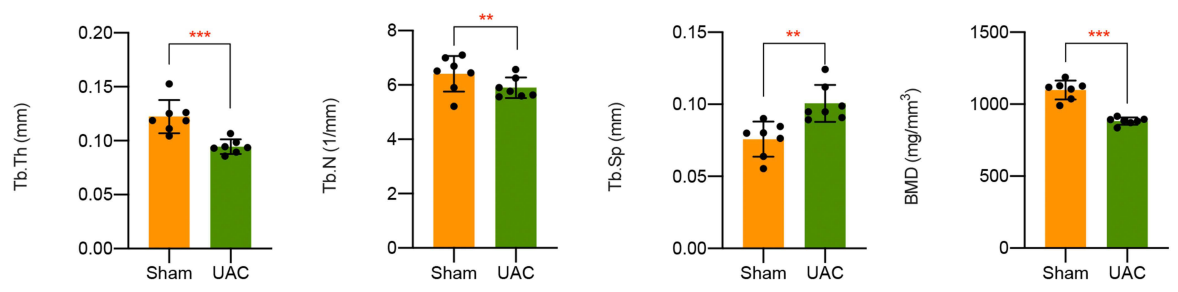

B

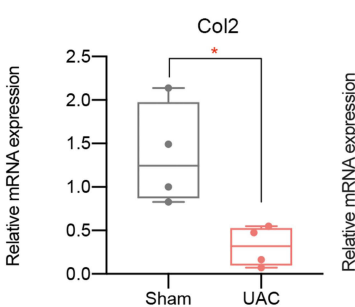

C
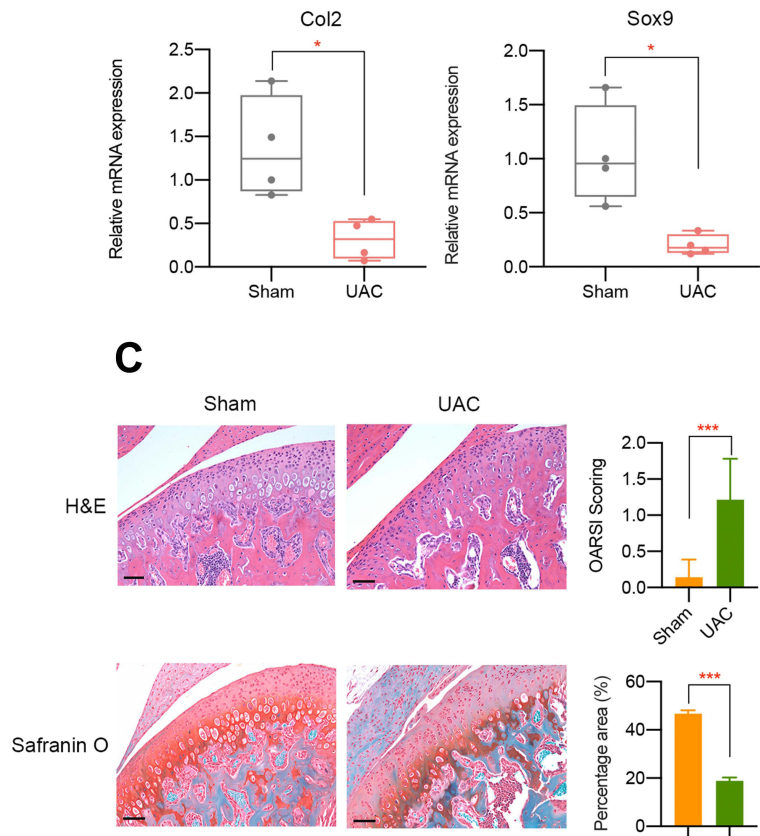

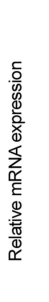
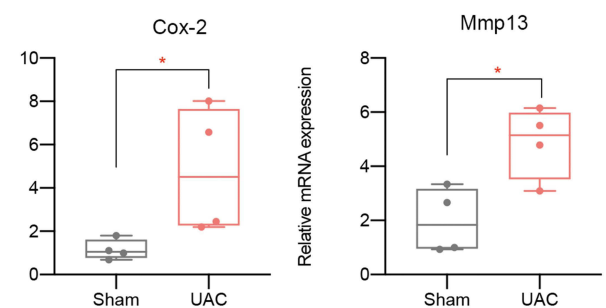

D
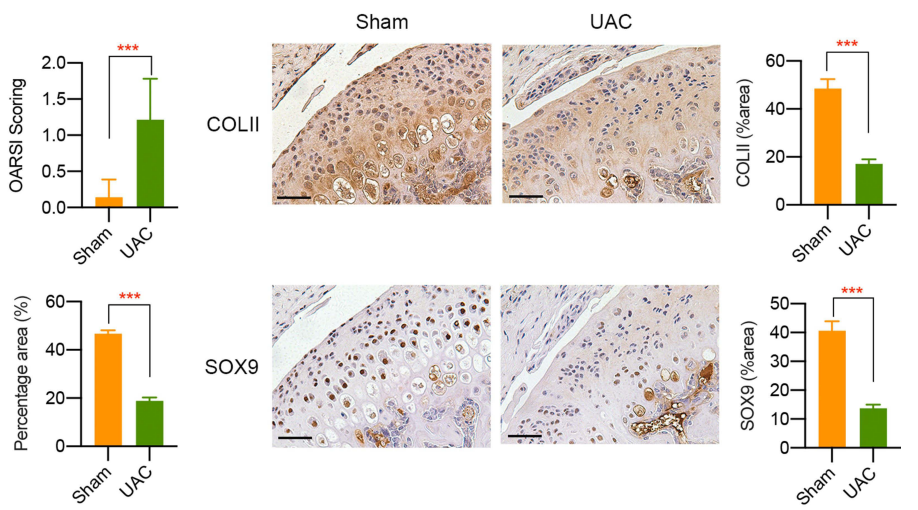
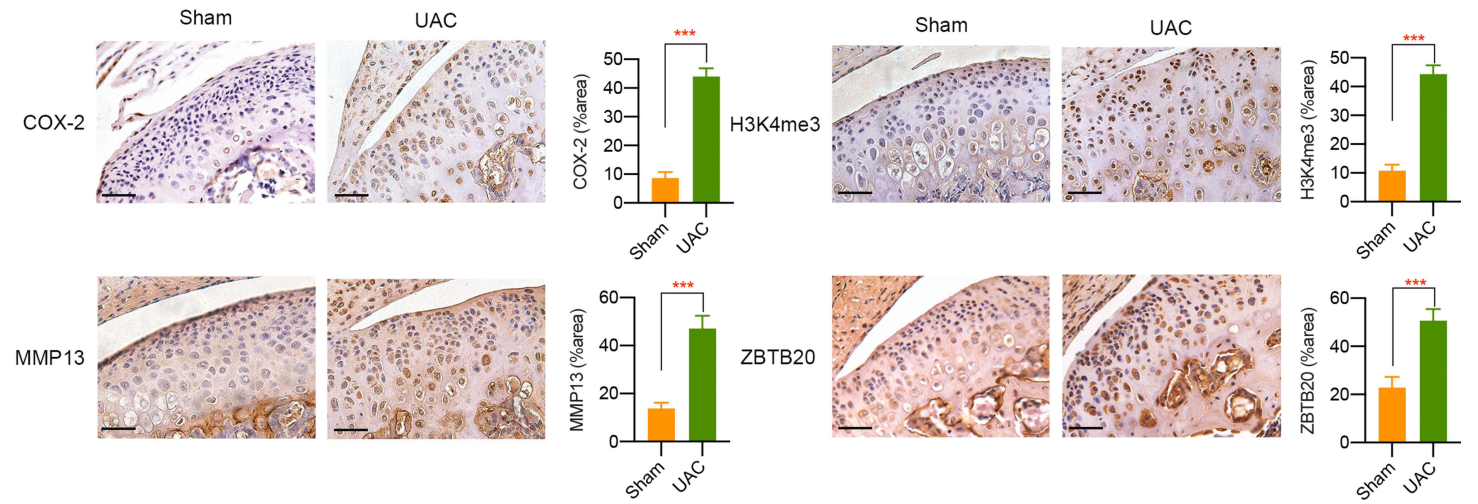

Figure 7 In vivo UAC mice model validation. (A) Micro-CT analysis of TMJ tissues from UAC group or sham controls. (B) mRNA level of Col2, Sox9, Cox-2, and Mmp/ 3 in TMJ samples in different groups was determined by qPCR. (C) H\&E and Safranin $O$ fast green staining of TMJ tissues from healthy control and UAC mice. (D) Immunohistochemistry assay of TMJ tissues from healthy control and UAC mice. Scar bar, $50 \mu \mathrm{m} . *_{\mathrm{p}}<0.05, *^{* *}<0.01, * * * \mathrm{p}<0.001$. 


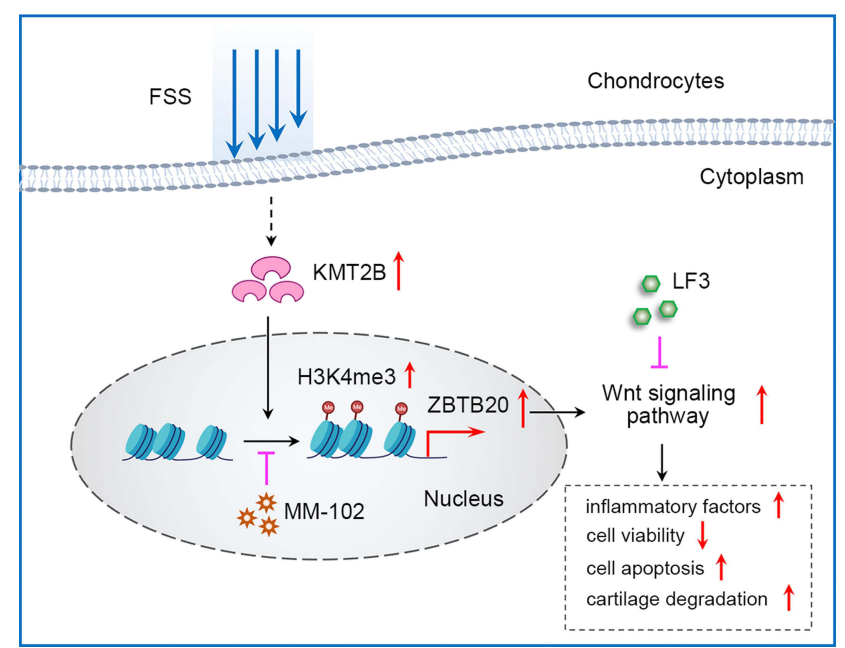

Figure 8 The overall working model of epigenetics-based mechanism underlying aberrant FSS-induced OA.

regulatory functions of $\mathrm{H} 3 \mathrm{~K} 4 \mathrm{me} 3-\mathrm{ZBTB} 20$ axis in shear stress-related TMJ pathogenesis. The overall working model of epigenetics-based mechanism underlying aberrant FSS-induced OA is illustrated in Figure 8.

\section{Discussion}

$\mathrm{OA}$ is a complicated disorder which involves the whole joint, including cartilage, subchondral bone, synovial membrane, meniscus, and infrapatellar fat pad. ${ }^{29-31}$ Emerging clinical treatment methods have limited effects that merely restricted to symptom relief. Recently, more and more studies have emphasized the importance of abnormal mechanical stress in the development and progression of OA. Mechanical environment was illustrated to play significant roles in regulating cell biological activities and maintaining homeostasis. ${ }^{32}$ Moreover, aberrant mechanical force may disturb cells and result in unintended responses. For example, shear stress was found to cause substantial changes in osteoblast cytoskeleton and nucleus morphology and volume, accompanied by adhesion and structural proteins changes. ${ }^{33}$ Furthermore, excessive high FSS in vivo could cause injury to BMSCs and hamper its attachment to the valvular scaffold. ${ }^{34}$ Another study demonstrated that histone methyltransferase EZH2 was decreased in response to FSS and endothelial gene expression was altered to result in a state of cell quiescence. ${ }^{35}$ Similarly, multiple studies focused on chondrocytes indicated the associations between aberrant mechanical stimulation and cartilage pathogenesis. It was suggested that relatively low FSS $\left(2-10\right.$ dynes $\left./ \mathrm{cm}^{2}\right)$ is chondroprotective while high FSS (10-20 dynes $\left./ \mathrm{cm}^{2}\right)$ has negative impact on chondrocytes, including inflammatory responses, cell apoptosis and death, and cartilage degradation. ${ }^{36}$ In the current study, we applied in vitro FSS model and our results indicated the negative impact of aberrant high FSS on primary chondrocytes, which was in compliance with previous studies. We made a relative comprehensive analysis of cell morphology, cell proliferation, inflammatory mediators production, cartilage degeneration and degradation under FSS treatment. Overall, aberrant high FSS has important regulatory functions on cartilage pathogenesis.

Given the potential regulatory mechanism in FSSinduced chondrocytes destruction, a study showed that high FSS induced MMP-12 expression via cyclic (c) AMP- and PGF2 $\alpha$-dependent signaling pathways and suggested critical roles of MMP-12 upregulation during the onset of OA. ${ }^{37}$ Another study illustrated an obvious activation of PI3K, ERK1/2, and JNK signaling, all may contribute to MMP-9 overexpression. ${ }^{38}$ Toll-like receptor 4 and caveolin- 1 were demonstrated to be binding partners in chondrocytes and their expression could be temporally regulated by FSS via upregulating PGE synthase-1 and L-PGDS. $^{39}$ All the above mechanistic investigation presented innovative insights for developing therapeutic strategies for cartilage-related diseases.

Recent evidences have indicated that epigenetic dysregulation was closely involved in OA pathogenesis, providing a novel mechanistic and therapeutic target. ${ }^{40}$ Specifically, one study demonstrated that EZH2 could modulate the histone methylation level in the promoter regions of miR-138 so as to influence cartilage degeneration. ${ }^{17}$ Another study found the crucial mediatory roles of HDAC in OA progression and suggested utilizing HDAC inhibitors to protect cartilage from disease. ${ }^{15}$ Moreover, SIRT1 downregulation was observed in human articular chondrocytes and its status was closely related with OA-like gene expression profiles, suggesting using SIRT1 as useful tool to protect against OA. ${ }^{41}$ Overall, the above results emphasized the importance of epigenetic regulators in joint diseases, which may serve as pharmacological candidates. Although epigenetic regulation has been implicated in OA, the specific regulatory mechanisms of high FSS on cartilage degeneration and degradation remain poorly defined. Our study firstly illustrated the regulation of histone methylation underlying shear stress-related OA and demonstrated a dramatic activation of $\mathrm{H} 3 \mathrm{~K} 4 \mathrm{me} 3$ in chondrocytes exposed to FSS treatment. Through the integrated analysis of transcriptome 
sequencing and H3K4me3 CUT\&Tag sequencing, we confirmed ZBTB20 as the direct target of H3K4me3 in FSSmediated OA pathogenesis.

ZBTB20 is a transcriptional repressor with multiple functions, such as regulating inflammation, cell death, immunity and so on. ${ }^{42,43}$ A study indicated that mice lacking ZBTB20 displayed abnormal growth rate, accompanied by high fatality and infertility, emphasizing the importance of ZBTB20 in organisms. ${ }^{44}$ In another study, ZBTB20 knockout could effectively protect mice from endotoxin shock and sepsis and further analysis showed that ZBTB20 may function through promoting NF- $\mathrm{kB}$ activation. ${ }^{45}$ What is more, ZBTB20 knockdown could alleviate proinflammatory factors produced by macrophages, transforming macrophages from M1 to M2 polarization, implying its essential roles in mediating inflammatory reactions. ${ }^{45}$ One study found that chondrocyte-specific ZBTB20 knockout mice presented delayed endochondral ossification and postnatal growth retardation. In depth investigation illustrated ZBTB20 could directly repress SOX9 expression to regulate hypertrophic chondrocytes differentiation. ${ }^{46}$ In line with these findings, the present study identified ZBTB20 as a critical modulator in the responses of chondrocytes to aberrant FSS disturbance, mainly manifested by inducing inflammatory responses and repressing cartilage-related markers. Further investigation illuminated the modulatory functions of ZBTB20 on chondrocytes were achieved by targeting Wnt pathway. We validated that Wnt signaling displayed overactivation in FSS-treated chondrocytes and Wnt inhibition could effectively alleviate FSS-induced destructive impact, which were consistent with former findings that excessive activation of Wnt signaling may lead to progressive joint damage. ${ }^{47,48}$ Given in vivo validation, we adopted UAC model by which an overexpression of H3K4me3 and ZBTB20 was evidently presented in UAC mice. It is a widely acknowledged animal model by which osteoarthritis-like cartilage degeneration in the mandibular condyles could be induced through imposing unilateral anterior crossbites on mice or rats..$^{22,23,28,49,50}$ In this study, we chose this model because it reflects aberrant stress-induced OA pathogenesis more naturally instead of injury-caused pathology changes. To get a more comprehensive understanding of mechanical-related OA, we may consider other OA models to validate our findings in the future.

In summary, we revealed novel epigenetic modifications during high FSS-related cartilage pathogenesis and identified H3K4me3-ZBTB20 as critical mediators Pharmacological inhibition of H3K4me3 or Wnt signaling could partially rescue the destructive effect of FSS on chondrocytes. To our knowledge, our findings firstly uncovered the potential relationship between mechanical stress and epigenetic events during OA pathogenesis, which could lay a foundation on discovering epigeneticsbased remedies for joint diseases.

\section{Abbreviations}

OA, osteoarthritis; FSS, fluid shear stress; UAC, unilateral anterior crossbites; SNP, sodium nitroprusside; DEGs, differentially expressed genes; EDTA, ethylenediaminetetraacetic acid; H\&E, hematoxylin-eosin; TMJOA, temporomandibular joint arthritis; TMJ, temporomandibular joint.

\section{Ethics Approval}

This study was approved by the Ethics Committee of Shanghai Jiao Tong University. All the animal studies were approved by the Shanghai Jiao Tong University Institute Animal Care and Use Committee, and conducted according to the Guide for Care and Use of Laboratory Animals (Ministry of Science and Technology of China, 2006).

\section{Acknowledgments}

This work was supported by National Natural Science Foundation of China (11932012, 81870790 and 31801233), Science and Technology Commission of Shanghai Municipality (19441906200 and 18441903600), Shanghai Rising-Star Program (19QA1405200), Innovative Research Team of High-level Local Universities in Shanghai (SSMU-ZDCX20180902), Young Doctor Collaborative Innovation Team of Ninth People's Hospital Affiliated to Shanghai Jiao Tong University, School of Medicine (QC2018-02) and Fundamental research program funding of Ninth People's Hospital affiliated to Shanghai Jiao Tong University School of Medicine (JYZZ085B).

\section{Author Contributions}

All authors made a significant contribution to the work reported, whether that is in the conception, study design, execution, acquisition of data, analysis and interpretation, or in all these areas; took part in drafting, revising or critically reviewing the article; gave final approval of the version to be published; have agreed on the journal to 
which the article has been submitted; and agree to be accountable for all aspects of the work.

\section{Disclosure}

The authors declare that there is no conflict of interests.

\section{References}

1. Englund M, Roemer FW, Hayashi D, Crema MD, Guermazi A. Meniscus pathology, osteoarthritis and the treatment controversy. Nat Rev Rheumatol. 2012;8(7):412-419. doi:10.1038/nrrheum.2012.69

2. Martel-Pelletier J, Barr AJ, Cicuttini FM, et al. Osteoarthritis. Nat Rev Dis Primers. 2016;2:16072. doi:10.1038/nrdp.2016.72

3. Hunter DJ, Schofield D, Callander E. The individual and socioeconomic impact of osteoarthritis. Nat Rev Rheumatol. 2014;10 (7):437-441. doi:10.1038/nrrheum.2014.44

4. Guilak F. Biomechanical factors in osteoarthritis. Best Pract Res Clin Rheumatol. 2011;25(6):815-823. doi:10.1016/j.berh.2011.11.013

5. Yokota H, Goldring MB, Sun HB. CITED2-mediated regulation of MMP-1 and MMP-13 in human chondrocytes under flow shear. $J$ Biol Chem. 2003;278(47):47275-47280. doi:10.1074/jbc.M304652200

6. Buckwalter JA, Martin JA, Brown TD. Perspectives on chondrocyte mechanobiology and osteoarthritis. Biorheology. 2006;43(34):603-609.

7. Kerin A, Patwari P, Kuettner K, Cole A, Grodzinsky A. Molecular basis of osteoarthritis: biomechanical aspects. Cell Mol Life Sci. 2002;59(1):27-35. doi:10.1007/s00018-002-8402-1

8. Healy ZR, Lee NH, Gao X, et al. Divergent responses of chondrocytes and endothelial cells to shear stress: cross-talk among COX-2, the Phase 2 response, and apoptosis. Proc Natl Acad Sci USA. 2005;102(39):14010-14015. doi:10.1073/pnas.0506620102

9. Carter DR, Wong M. Modelling cartilage mechanobiology. Philos Trans $R$ Soc Lond B Biol Sci. 2003;358(1437):1461-1471. doi:10.1098/rstb.2003.1346

10. Carter DR, Beaupre GS, Wong M, Smith RL, Andriacchi TP, Schurman DJ. The mechanobiology of articular cartilage development and degeneration. Clin Orthop Relat Res. 2004;427(427 Suppl): S69-77. doi:10.1097/01.blo.0000144970.05107.7e

11. Wang P, Guan PP, Guo C, Zhu F, Konstantopoulos K, Wang ZY. Fluid shear stress-induced osteoarthritis: roles of cyclooxygenase-2 and its metabolic products in inducing the expression of proinflammatory cytokines and matrix metalloproteinases. FASEB J. 2013;27 (12):4664-4677. doi:10.1096/fj.13-234542

12. Hunziker EB. Articular cartilage repair: basic science and clinical progress. A review of the current status and prospects. Osteoarthritis Cartilage. 2002;10(6):432-463.

13. Kan RL, Chen J, Sallam T. Crosstalk between epitranscriptomic and epigenetic mechanisms in gene regulation. Trends Genet. 2021. doi:10.1016/j.tig.2021.06.014

14. Yuan G, Flores NM, Hausmann S, et al. Elevated NSD3 histone methylation activity drives squamous cell lung cancer. Nature. 2021;590(7846):504-508. doi:10.1038/s41586-020-03170-y

15. Zhang H, Ji L, Yang Y, Zhang X, Gang Y, Bai L. The role of HDACs and HDACi in cartilage and osteoarthritis. Front Cell Dev Biol. 2020;8:560117. doi:10.3389/fcell.2020.560117

16. Wen ZH, Huang JS, Lin YY, et al. Chondroprotective effects of a histone deacetylase inhibitor, panobinostat, on pain behavior and cartilage degradation in anterior cruciate ligament transection-induced experimental osteoarthritic rats. Int $J$ Mol Sci. 2021;22:14. doi:10.3390/ijms22147290

17. Wang J, Wang X, Ding X, Huang T, Song D, Tao H. EZH2 is associated with cartilage degeneration in osteoarthritis by promoting SDC1 expression via histone methylation of the microRNA-138 promoter. $L a b$ Invest. 2021;101(5):600-611. doi:10.1038/s41374-021-00532-6
18. Yang D, Okamura H, Nakashima Y, Haneji T. Histone demethylase Jmjd3 regulates osteoblast differentiation via transcription factors Runx2 and osterix. J Biol Chem. 2013;288(47):33530-33541. doi:10.1074/jbc. M113.497040

19. Ye L, Fan Z, Yu B, et al. Histone demethylases KDM4B and KDM6B promote osteogenic differentiation of human MSCs. Cell Stem Cell. 2018;23(6):898-899. doi:10.1016/j.stem.2018.11.002

20. Kaya-Okur HS, Wu SJ, Codomo CA, et al. CUT\&Tag for efficient epigenomic profiling of small samples and single cells. Nat Commun. 2019;10(1):1930. doi:10.1038/s41467-019-09982-5

21. Lu L, Zhang X, Zhang M, et al. RANTES and SDF-1 are keys in cell-based therapy of TMJ osteoarthritis. J Dent Res. 2015;94 (11):1601-1609. doi:10.1177/0022034515604621

22. Liu YD, Liao LF, Zhang HY, et al. Reducing dietary loading decreases mouse temporomandibular joint degradation induced by anterior crossbite prosthesis. Osteoarthritis Cartilage. 2014;22 (2):302-312. doi:10.1016/j.joca.2013.11.014

23. Weng Y, Liu Y, Du H, et al. Glycosylation of DMP1 is essential for chondrogenesis of Condylar cartilage. J Dent Res. 2017;96 (13):1535-1545. doi:10.1177/0022034517717485

24. Bernstein BE, Kamal M, Lindblad-Toh K, et al. Genomic maps and comparative analysis of histone modifications in human and mouse. Cell. 2005;120(2):169-181. doi:10.1016/j.cell.2005.01.001

25. Cherifi C, Monteagudo S, Lories RJ. Promising targets for therapy of osteoarthritis: a review on the Wnt and TGF-beta signalling pathways. Ther Adv Musculoskelet Dis. 2021;13:1759720X211006959. doi:10.1177/ 1759720X211006959

26. Lories RJ, Monteagudo S. Review article: is Wnt signaling an attractive target for the treatment of osteoarthritis? Rheumatol Ther. 2020;7 (2):259-270. doi:10.1007/s40744-020-00205-8

27. Sun JL, Yan JF, Yu SB, Zhao J, Lin QQ, Jiao K. MicroRNA-29b promotes subchondral bone loss in TMJ osteoarthritis. J Dent Res. 2020;99(13):1469-1477. doi:10.1177/0022034520937617

28. Zhang J, Liao L, Zhu J, et al. Osteochondral interface stiffening in mandibular Condylar osteoarthritis. J Dent Res. 2018;97(5):563-570. doi: $10.1177 / 0022034517748562$

29. Mathiessen A, Conaghan PG. Synovitis in osteoarthritis: current understanding with therapeutic implications. Arthritis Res Ther. 2017;19(1):18. doi:10.1186/s13075-017-1229-9

30. Belluzzi E, Macchi V, Fontanella CG, et al. Infrapatellar fat pad gene expression and protein production in patients with and without osteoarthritis. Int J Mol Sci. 2020;21:17. doi:10.3390/ijms21176016

31. Donell S. Subchondral bone remodelling in osteoarthritis. EFORT Open Rev. 2019;4(6):221-229. doi:10.1302/2058-5241.4.180102

32. Adams MA. The mechanical environment of chondrocytes in articular cartilage. Biorheology. 2006;43(3-4):537-545.

33. Jin J, Jaspers RT, Wu G, Korfage JAM, Klein-Nulend J, Bakker AD. Shear stress modulates osteoblast cell and nucleus morphology and volume. Int J Mol Sci. 2020;21:21. doi:10.3390/ijms21218361

34. Ye X, Zhao Q, Sun X, Li H. Enhancement of mesenchymal stem cell attachment to decellularized porcine aortic valve scaffold by in vitro coating with antibody against CD90: a preliminary study on antibody-modified tissue-engineered heart valve. Tissue Eng Part A. 2009;15(1):1-11. doi:10.1089/ten.tea.2008.0001

35. Maleszewska M, Vanchin B, Harmsen MC, Krenning G. The decrease in histone methyltransferase EZH2 in response to fluid shear stress alters endothelial gene expression and promotes quiescence. Angiogenesis. 2016;19(1):9-24. doi:10.1007/s10456-015-9485-2

36. Chang SF, Huang KC, Chang HI, Lee KC, Su YP, Chen CN. 2 dyn/cm(2) shear force upregulates kruppel-like factor 4 expression in human chondrocytes to inhibit the interleukin-1beta-activated nuclear factor-kappaB. J Cell Physiol. 2018;234(1):958-968. doi:10.1002/jcp.26924

37. Guan PP, Ding WY, Wang P. The roles of prostaglandin F2 in regulating the expression of matrix metalloproteinase-12 via an insulin growth factor-2-dependent mechanism in sheared chondrocytes. Signal Transduct Target Ther. 2018;3:27. doi:10.1038/s41392-018-0029-2 
38. Wang P, Zhu F, Konstantopoulos K. The antagonistic actions of endogenous interleukin-1beta and 15-deoxy-Delta12,14prostaglandin J2 regulate the temporal synthesis of matrix metalloproteinase-9 in sheared chondrocytes. $J$ Biol Chem. 2012;287(38):31877-31893. doi:10.1074/jbc.M112.362731

39. Wang P, Zhu F, Tong Z, Konstantopoulos K. Response of chondrocytes to shear stress: antagonistic effects of the binding partners Toll-like receptor 4 and caveolin-1. FASEB J. 2011;25 (10):3401-3415. doi:10.1096/fj.11-184861

40. Grandi FC, Bhutani N. Epigenetic Therapies for Osteoarthritis. Trends Pharmacol Sci. 2020;41(8):557-569. doi:10.1016/j.tips.2020.05.008

41. Fujita N, Matsushita T, Ishida K, et al. Potential involvement of SIRT1 in the pathogenesis of osteoarthritis through the modulation of chondrocyte gene expressions. J Orthop Res. 2011;29(4):511-515. doi:10.1002/jor.21284

42. Sun Y, Preiss NK, Valenteros KB, et al. Zbtb20 restrains CD8 T cell immunometabolism and restricts memory differentiation and antitumor immunity. J Immunol. 2020;205(10):2649-2666. doi:10.4049/ jimmunol.2000459

43. Ren AJ, Wang K, Zhang H, et al. ZBTB20 regulates nociception and pain sensation by modulating TRP channel expression in nociceptive sensory neurons. Nat Commun. 2014;5:4984. doi:10.1038/ncomms5984

44. Nagao M, Ogata T, Sawada Y, Gotoh Y. Zbtb20 promotes astrocytogenesis during neocortical development. Nat Commun. 2016;7:11102. doi:10.1038/ncomms11102
45. Liu X, Zhang P, Bao Y, et al. Zinc finger protein ZBTB20 promotes toll-like receptor-triggered innate immune responses by repressing IkappaBalpha gene transcription. Proc Natl Acad Sci U S A. 2013;110(27):11097-11102. doi:10.1073/pnas.1301257110

46. Zhou G, Jiang X, Zhang H, et al. Zbtb20 regulates the terminal differentiation of hypertrophic chondrocytes via repression of Sox9. Development. 2015;142(2):385-393. doi:10.1242/dev.108530

47. Lories RJ, Peeters J, Bakker A, et al. Articular cartilage and biomechanical properties of the long bones in Frzb-knockout mice. Arthritis Rheum. 2007;56(12):4095-4103. doi:10.1002/art.23137

48. Zhu M, Tang D, Wu Q, et al. Activation of beta-catenin signaling in articular chondrocytes leads to osteoarthritis-like phenotype in adult beta-catenin conditional activation mice. J Bone Miner Res. 2009;24 (1):12-21. doi:10.1359/jbmr.080901

49. Sun JL, Yan JF, Li J, et al. Conditional deletion of Adrb2 in mesenchymal stem cells attenuates osteoarthritis-like defects in temporomandibular joint. Bone. 2020;133:115229. doi:10.1016/j.bone.2020.11 5229

50. Liu Q, Yang $\mathrm{H}$, Zhang $\mathrm{M}$, et al. Initiation and progression of dental-stimulated temporomandibular joints osteoarthritis. Osteoarthritis Cartilage. 2021;29(5):633-642. doi:10.1016/j.joca.20 20.12.016
Journal of Inflammation Research

\section{Publish your work in this journal}

The Journal of Inflammation Research is an international, peerreviewed open-access journal that welcomes laboratory and clinical findings on the molecular basis, cell biology and pharmacology of inflammation including original research, reviews, symposium reports, hypothesis formation and commentaries on: acute/chronic inflammation; mediators of inflammation; cellular processes; molecular

\section{Dovepress}

mechanisms; pharmacology and novel anti-inflammatory drugs; clinical conditions involving inflammation. The manuscript management system is completely online and includes a very quick and fair peerreview system. Visit http://www.dovepress.com/testimonials.php to read real quotes from published authors. 